Mens

revue d'histoire intellectuelle de l'Amérique française

MENS

\title{
Une réconciliation insaisissable : le mouvement de la bonne entente, 1916-1930
}

\section{Robert Talbot}

Volume 8, numéro 1, automne 2007

URI : https://id.erudit.org/iderudit/1023148ar

DOI : https://doi.org/10.7202/1023148ar

Aller au sommaire du numéro

\section{Éditeur(s)}

Centre de recherche en civilisation canadienne-française

ISSN

1492-8647 (imprimé)

1927-9299 (numérique)

Découvrir la revue

Citer cet article

Talbot, R. (2007). Une réconciliation insaisissable : le mouvement de la bonne entente, 1916-1930. Mens, 8(1), 67-125. https://doi.org/10.7202/1023148ar

\section{Résumé de l'article}

Le mouvement de la « bonne entente » (1916 - années 1930), lancé par un petit groupe d'anglophones ontariens, avait pour objectif de favoriser le dialogue entre Canadiens français et Canadiens anglais, qui faisait cruellement défaut pendant la Première Guerre mondiale, par le rapprochement entre les élites des deux groupes linguistiques. Jusqu'ici, les historiens ont surtout vu dans ce mouvement une stratégie visant à faire accepter en douce la conscription aux Canadiens français. Dans cet article, nous soutenons toutefois que la conscription n'était pas nécessairement la seule priorité, ni même la plus importante, dans l'esprit de nombreux membres anglophones. L'objectif de la Ligue de la Bonne Entente (1916) était plutôt de retrouver l'esprit d'unité et de ferveur patriotique qui avait imprégné la société canadienne - française et anglaise - au moment du déclenchement des hostilités. Après la guerre, la Ligue et les organisations qui lui ont succédé ont exigé, en coulisse, l'abrogation du Règlement XVII par le gouvernement de l'Ontario. De la Première Guerre mondiale à la crise, le mouvement de la bonne entente a prêché modération et unité dans une société très divisée. Il a contribué à rétablir le processus de rapprochement des élites et a donné naissance à un nationalisme civique canadien.
Ce document est protégé par la loi sur le droit d'auteur. L'utilisation des services d'Érudit (y compris la reproduction) est assujettie à sa politique d'utilisation que vous pouvez consulter en ligne.

https://apropos.erudit.org/fr/usagers/politique-dutilisation/ 


\title{
UNE RÉCONCILIATION INSAISISSABLE : LE MOUVEMENT DE LA BONNE ENTENTE, 1916-1930
}

\author{
Robert Talbot \\ Département d'histoire \\ Université d'Ottawa
}

\section{Résumé}

Le mouvement de la «bonne entente » (1916 - années 1930), lancé par un petit groupe d'anglophones ontariens, avait pour objectif de favoriser le dialogue entre Canadiens français et Canadiens anglais, qui faisait cruellement défaut pendant la Première Guerre mondiale, par le rapprochement entre les élites des deux groupes linguistiques. Jusqu'ici, les historiens ont surtout vu dans ce mouvement une stratégie visant à faire accepter en douce la conscription aux Canadiens français. Dans cet article, nous soutenons toutefois que la conscription n'était pas nécessairement la seule priorité, ni même la plus importante, dans l'esprit de nombreux membres anglophones. L'objectif de la Ligue de la Bonne Entente (1916) était plutôt de retrouver l'esprit d'unité et de ferveur patriotique qui avait imprégné la société canadienne - française et anglaise - au moment du déclenchement des hostilités. Après la guerre, la Ligue et les organisations qui lui ont succédé ont exigé, en coulisse, l'abrogation du Règlement XVII par le gouvernement de l'Ontario. De la Première Guerre mondiale à la crise, le mouvement de la bonne entente a prêché modération et unité dans une société très divisée. Il a contribué à rétablir le processus de rapprochement des élites et a donné naissance à un nationalisme civique canadien. 


\section{Abstract}

The "bonne entente" movement (1916-1930s), initiated by a small group of Ontario anglophones, began as an exercise in elite accommodation with the purpose of fostering a friendly dialogue between English-and French-speaking Canadians that was sorely lacking during the Great War years. In attempting to explain "bonne entente" within the confines of the traditional narrative, previous authors have described how the movement's anglophone membership used it to soft-peddle their francophone counterparts into accepting conscription. It is argued here, bowever, that recruitment and conscription were not necessarily the sole nor the first priority in the minds of many anglophone members. Instead; the creators of the Bonne Entente League (1916) were initially more intent on recapturing the spirit of unity and patriotic fervour that had permeated Canadian society French and British - at the wiar's outset. Indeed, the League and its successor organizations persisted into the post-war era to become a behind-the-scenes common front for the repeal of Ontario's discriminatory Regulation XVII against Frenchlanguage rights. From the Great War through to the Great Depression, the bonne entente movement provided a voice for moderation and unity in an otherwise divided society. It helped restore the process of elite accommodation and gave rise to a civic Canadian nationalism.

La période de la Grande Guerre est souvent considérée comme celle qui fut le plus source de division dans l'histoire intérieure du Canada. Selon les historiens, l'opposition canadienne-française à la conscription et, par moments, à la simple participation à la guerre suscita la colère des Canadiens anglophones impérialistes, qui étaient plutôt intransigeants. «Au cours de la guerre mondiale de 1914-1918, a résumé une historienne, le "nationalisme" canadien-français s'est mué en haine contre [...] les demandes d'hommes et d'argent pour pouvoir poursuivre la guerre. ${ }^{2}$ »

Même avant la guerre, les relations entre les deux solitudes du Canada avaient été caractérisées par le conflit entre l'impérialisme canadien-anglais et le nationalisme canadienfrançais. L'objectif des impérialistes canadiens-anglais était 
de « consolider l'empire britannique par des moyens militaires, économiques et constitutionnels ». Comme l'explique Carl Berger, «l'impérialisme était une forme de nationalisme canadien ». Des hommes tels que George Munro Grant et le colonel George Taylor Denison « croyaient que le Canada ne pouvait obtenir le statut de nation qu'en maintenant ses liens avec l'Empire et en acquérant de l'influence au sein de ses conseils ». Il était donc essentiel que le Canada joue un rôle significatif dans les guerres impériales, et particulièrement la Grande Guerre. En outre, le «nouvel impérialisme » de la fin du XIX ${ }^{e}$ siècle avait une connotation davantage raciale et était beaucoup moins tolérant que l'impérialisme mercantile qui l'avait précédé. Les impérialistes canadiens anglophones les plus intransigeants, comme l'orangiste D'Alton McCarthy, avaient utilisé le discours impérialiste pour promouvoir un programme d'assimilation et d'attaque contre les minorités francophones hors du Québec. Sans surprise, les Canadiens français en vinrent à considérer l'impérialisme canadien-anglais « avec une très grande hostilité ${ }^{3}$ ». Contrairement à leurs homologues anglophones, ils n'étaient guère attachés sentimentalement à l'Empire britannique. Des nationalistes tels que Henri Bourassa voyaient dans l'impérialisme britannique et les guerres étrangères une menace à l'unité nationale. Il préconisait un Canada plus autonome où la nature bilingue du pays serait davantage appréciée 4 .

On a beaucoup glosé sur ce qui séparait les Canadiens d'origines britannique et française tout particulièrement au cours des années de la guerre, mais les tentatives de rapprochement et de réconciliation ont nettement moins intéressé les historiens. En août 1916, par exemple, au milieu de la période de bouleversements que fut la Première Guerre mondiale, un petit groupe de politiciens et d'hommes d'affaires ontariens et québécois, libéraux et conservateurs, anglopho- 
nes et francophones, se réunit à Montréal pour déclarer l'importance qu'il accordait à la grande cause de l'unité nationale.

Nous, Canadiens de langues française et anglaise, réunis pour la promotion de l'unité nationale, ayant établi par des échanges amicaux un respect mutuel et nous étant fermement convaincus de l'impartialité innée de la grande majorité des deux races, affirmons par la présente croire inaltérablement qu'il n'y a pas maintenant, et qu'il n'y aura jamais, de problème entre les deux races du Canada qui ne puisse, et, de plein droit, ne doive être réglé à l'amiable et équitablement, et de manière à donner satisfaction à la grande majorité de ceux qui sont concernés 5 .

C'est ainsi que naquit le mouvement de la bonne entente, dont le nom s'inspirait de l'«entente cordiale » du temps de guerre entre les deux mères patries d'outre-Atlantique. La Ligue de la Bonne Entente voulait réunir des personnalités des communautés anglophones et francophones désunies de l'Ontario et du Québec dans une atmosphère cordiale, contribuant à établir des relations amicales et une compréhension mutuelle. Les participants étaient surtout des professionnels et des politiciens des classes moyennes ou supérieures, de l'Ontario ou du Québec, et, jusqu'aux dernières années du mouvement, il s'agissait presque exclusivement d'hommes.

L'objectif officiel du mouvement de la bonne entente était toujours de favoriser l'unité nationale, mais le fait que ses membres étaient d'origines diverses lui donnait des objectifs secondaires également divers. La séparation la plus évidente s'observait entre les Canadiens anglophones surtout protestants et les Canadiens français catholiques. Des politiciens nationalistes tels que le député libéral fédéral Paul-Émile Lamarche se servirent de la «bonne entente » pour exprimer leur désapprobation du statut colonial du Canada au sein de 
l'Empire, et pour dénoncer des pratiques de recrutement militaire malhonnêtes. Des orangistes tels que le député conservateur fédéral J. W. Edwards, à l'opposé, se servaient du mouvement pour défendre des politiques linguistiques discriminatoires et appeler à l'assimilation des francophones. Ces divisions se trouvaient compliquées par les intérêts professionnels divers des participants. Le mouvement en vint à inclure un éventail de politiciens de tous les partis, et des trois ordres de gouvernement, ainsi que divers avocats, universitaires, médecins, ecclésiastiques, journalistes, hommes d'affaires, industriels, recruteurs des forces armées et militaires. Pour certains politiciens, le mouvement constituait une plate-forme leur assurant une plus grande visibilité - un moyen de faire de la publicité pour leur propre personne et leur parti. Les hommes d'affaires et les industriels souhaitaient souvent davantage consolider leurs intérêts commerciaux dans une province ou l'autre, plutôt que favoriser l'unité nationale. Les recruteurs des forces armées espéraient qu'en améliorant les relations entre francophones et anglophones, ils pourraient atténuer l'opposition à l'enrôlement au Québec et éviter ainsi la conscription. Quelques militaires, de leur côté, voulaient se servir de la bonne entente pour exiger une approbation sans détour de la conscription. Quels que soient ces motifs parallèles, une bonne proportion des bonne-ententistes demeurait motivée par un désir sincère d'empêcher le pays de se désagréger.

On peut dire que le mouvement de la bonne entente est passé par trois phases différentes au cours de son existence, de 1916 aux années 1930. D’abord, la création et les premières activités de la Ligue de la Bonne Entente originelle, en 1916 et 1917. Cette phase débuta au beau milieu des débats houleux sur la conscription et du conflit sur les droits des francophones en Ontario. Elle fut marquée par un 
optimisme initial, suivi d'une désillusion, le pays demeurant divisé sur ces questions. À la fin de la guerre, la deuxième phase du mouvement de la bonne entente ${ }^{6}$, de 1918 à 1927 , fut marquée par le retour à un discours plus franc et le retrait du Règlement XVII discriminatoire (qui limitait sérieusement l'enseignement en langue française dans les écoles ontariennes), en 1927. Le mouvement fut revigoré par l'arrivée de nouveaux membres, dont des universitaires et des Franco-Ontariens, ainsi que par la fondation de nouvelles organisations, c'est-à-dire la Better Understanding Association (1918) et l'influente Unity League of Ontario (1921). La troisième et dernière phase du mouvement de la bonne entente fut marquée par la disparition de sa cause célèbre - l'abrogation du Règlement XVII - et par le début de la Crise. Du milieu des années 1920 aux années 1930, le mouvement de la bonne entente perdit graduellement de sa pertinence, et finit par disparaître. De récents auteurs ont abordé ces différentes phases isolément les unes des autres, mais le présent texte se veut une analyse plus générale portant sur le mouvement dans son ensemble.

Le mouvement de la bonne entente n'a bien évidemment guère attiré l'attention des historiens - son existence même se prête mal au récit historique traditionnel. Bien sûr, on en fait souvent mention en passant dans les manuels et dans les histoires générales portant sur ce que fut la Grande Guerre pour le Canada. Les auteurs de ces textes tentent généralement de l'intégrer au thème plus global de l'animosité entre Canada français et Canada anglais. Ce faisant, ils réduisent le mouvement à un outil déloyal en faveur de la conscription. Pour les anglophones, soutiennent-ils, l'unité nationale était une préoccupation secondaire, car ils tentaient de se servir de la « bonne entente » pour amadouer leurs homologues francophones et les amener à accepter la conscription. 
«En 1917, expliquaient Robert Craig Brown et Ramsay Cook, historiens renommés, la Bonne Entente avait échoué, et ses parrains canadiens-anglais en étaient venus à préconiser ouvertement la conscription et un gouvernement de coalition sous le nouveau nom de Win-the-War Movement. » Les auteurs décrivent les raisons de l'échec du mouvement :

[Un] mouvement dont les objectifs n'ont jamais été vraiment totalement partagés, voire peut-être compris, à la fois par les membres francophones et anglophones pouvait difficilement connaittre le succès de façon permanente. Les Canadiens français semblent avoir espéré que la «bonne entente » contribue à une solution satisfaisante de la question linguistique en Ontario ; les membres anglophones s'attendaient quant à eux à ce qu'elle incite leurs compatriotes francophones à s'enthousiasmer davantage pour la guerre?

Dans le même esprit, l'ouvrage récent d'Yvan Lamonde sur l'histoire intellectuelle du Québec fait figurer les mouvements de «Bonne Entente» parmi les campagnes de propagande visant à convaincre les francophones d'accepter le service obligatoire. Son chapitre sur la guerre porte plutôt sur les thèmes plus traditionnels de l'opposition des Canadiens français à la participation et de la crise de la conscription ${ }^{8}$.

L'article de Brian Cameron sur les deux premières années de la Ligue de la Bonne Entente aborde le thème de la duplicité canadienne-anglaise. Il soutient lui aussi que les participants anglophones n'avaient à l'esprit que la conscription ainsi que des intérêts commerciaux et politiques. Quand la Ligue est devenue la Win-the-War Convention, explique-t-il, les membres francophones du mouvement se sont sentis trompés et trahis ${ }^{9}$. Les Canadiens anglais n'avaient rien appris de leurs rencontres directes avec l'élite de la société canadiennefrançaise. Le gouvernement de coalition et la conscription étant 
assurés, ils abandonnèrent carrément la «bonne entente ». À l'instar de Cook, Brown et Lamonde, Cameron achève son examen du mouvement en 1917.

À l'opposé, des spécialistes franco-ontariens tels que Robert Choquette et Gaétan Gervais ont analysé les initiatives de l'après-guerre relatives à la «bonne entente». Leurs conclusions sont beaucoup plus favorables. Ils montrent comment les leaders franco-ontariens, sous la conduite du sénateur Napoléon Belcourt, se sont adressés à des anglophones bien disposés et influents du cœur orangiste de Toronto pour créer la Unity League of Ontario. Ce mouvement de pression, soutiennent-ils, servit à changer graduellement les attitudes au sein du gouvernement provincial et dans le public en général. Il rendit finalement possible l'abrogation du Règlement $\mathrm{XVII}^{10}$. Leur travail nous offre, ce qui est rare, un coup d'œil sur le mouvement de la bonne entente au cours des années 1920, mais il ne le place pas dans le contexte global de ses débuts en 1916-1917.

Le lecteur intéressé doit parcourir l'ouvrage monumental de Robert Rumilly sur l'histoire du Québec, achevé il y a une cinquantaine d'années, pour trouver une présentation à plus long terme du mouvement de la bonne entente. L'opinion de Rumilly sur la Ligue de la Bonne Entente originelle est proche de celle d'historiens ultérieurs : "Le voyage de "Bonne Entente" atténua peut-être les préjugés de quelques hommes d'affaires ontariens. Il n'eut pas d'autre résultat pratique. Il ne changea rien à la situation scolaire ${ }^{11}$. „ Dans son analyse des initiatives bonne-ententistes de la période d'aprèsguerre, Rumilly demeure tout aussi critique des motivations de plusieurs participants. Il reconnait néanmoins que les efforts du sénateur Belcourt et de la Unity League of Ontario ont contribué à orienter l'opinion ontarienne en faveur de l'abrogation du Règlement XVII. «Par le contact avec des 
Anglo-Caniadiens de ce genre - assez gentilshommes pour revenir sur des préjugés - Belcourt a obtenu, à la Unity League, des conversions décisives", explique-t-il ${ }^{12}$.

Une étude plus nuancée et plus complète du mouvement de la bonne entente, de ses débuts en 1916 à ses activités dans l'après-guerre, et à son déclin à la fin des années 1920 et pendant les années 1930, révèle une complexité que l'on ne discerne pas dans les analyses récentes. Premièrement, pour bien comprendre l'inspiration originelle et les objectifs de ses créateurs, on doit placer la création du mouvement dans le contexte plus général de l'esprit d'unité qui prévalait au sein de la société canadienne - anglophone et francophone - au début de la guerre. C'est cet esprit d'unité que les créateurs de la Ligue de la Bonne Entente recherchaient à l'origine, et non une approbation tacite de la conscription. Deuxièmement, une analyse plus systématique des divers objectifs et idées du mouvement par la suite nous rappelle que les participants francophones et anglophones du mouvement de la bonne entente ne formaient pas un groupe monolithique. La Ligue de 1916-1917 n'avait pas le même sens pour tout le monde, et pas forcément en fonction de la langue. Plus précisément, recrutement et conscription n'étaient pas nécessairement la seule, ni même la priorité dans l'esprit de certains membres anglophones. Finalement, si l'on va au-delà des premières années du mouvement, on constate que le mouvement de la bonne entente ne disparut pas simplement avec le mouvement Win-the-War. Au contraire, il survécut dans la période d'après-guerre pour devenir un forum plus significatif de débat, et, par la suite, un front commun dans les coulisses pour l'abrogation du Règlement XVII discriminatoire de l'Ontario. Au cours des dernières années, cependant, le mouvement de la bonne entente perdit de sa pertinence et fut finalement éclipsé par les difficultés plus urgentes des années 1930. 
L'importance globale du mouvement aux yeux du public demeure au mieux insaisissable. Mais tout au moins, il est resté important pour ceux qui ont défendu sa cause. Le présent article soutient que de la Grande Guerre à la crise, le mouvement de la bonne entente - sous ses diverses formes et compte tenu de son degré fluctuant d'influence - a continué de préconiser modération et unité dans une société autrement divisée. En outre, il a contribué à restaurer le processus de rapprochement des élites et a donné naissance à un nationalisme civique canadien.

\section{La Ligue de la Bonne Entente, 1916-1917}

Un examen du contexte de la création de la Ligue de la Bonne Entente permet de saisir l'inspiration et les objectifs initiaux du mouvement. «Au cours de l'été 1916 », commence l'histoire officielle du mouvement,

plusieurs Ontariens ont réalisé que si l'on ne faisait rien pour combler le fossé séparant les deux principales races du Canada, et particulièrement les relations entre les deux plus grandes provinces, l'unité nationale dans le Dominion risque d'être mise en péril et les bons sentiments résultant du déclenchement de la guerre pourraient disparaittre ${ }^{13}$.

Au début de la guerre, les Canadiens français avaient semblé aussi enthousiastes à l'égard de la guerre que leurs compatriotes anglophones, et pendant une brève période, les deux « peuples fondateurs » avaient semblé unis par cette cause. Même Henri Bourassa, nationaliste et plus tard symbole de l'opposition à la participation canadienne au conflit, "croyait alors que la guerre pourrait favoriser "l'union des races" anglaise et française au Canada ${ }^{14}$ ». Le Devoir, le quotidien de Bourassa, faisait état avec enthousiasme des foules qui avaient célébré à Québec les déclarations de guerre : 
La nouvelle officielle de la guerre entre l'Allemagne et la Grande-Bretagne a été reçue ici au milieu du plus grand enthousiasme : Canadiens français, Anglais, et Irlandais ont paradé côte à côte dans un même élan de loyauté et de patriotisme. [...] [L]es Canadiens français comprenaient tout le sérieux de la situation présente pour l'Empire et ils marcheraient épaule à épaule avec leurs concitoyens de races différentes ${ }^{15}$.

L'enthousiasme général du Canada français, écrit François Charbonneau, "s'explique par un désir de [...] prouver la valeur des siens aux yeux [des] compatriote[s] de langue anglaise $^{16} »$. Le désir de jouer un rôle important dans l'effort de guerre du Canada n'aurait pu être plus évident que lors d'une " réunion de recrutement monstre » en octobre 1914 au parc Sohmer, à Montréal, pour la création du $22^{\mathrm{e}}$ Bataillon (canadien-français). Sir Wilfrid Laurier, le chef libéral de l'opposition, le premier ministre provincial Lomer Gouin et même le chef de l'opposition provinciale se réunirent pour affirmer le devoir du Canada de défendre ses mères patries en Europe ${ }^{17}$.

Toutefois, le sentiment général apparent d'unité nationale céda bientôt la place à une animosité raciale profondément enracinée. La principale pomme de discorde entre $\mathrm{Ca}$ nadiens francophones et anglophones était le Règlement XVII. Imposé en 1912, il restreignait de façon importante l'enseignement en français dans les écoles ontariennes. L'historien Jean Provencher écrit : "[d]urant les premiers mois de 1915, le Québec s'intéresse plus à la question du Règlement XVII qu'à la guerre en Europe ${ }^{18}$. » Charbonneau explique la contradiction logique que le Règlement XVII posait aux Canadiens français relativement à l'enrôlement :

Le parallèle sera tracé rapidement entre les minorités européennes que l'Angleterre prétend défendre en Europe et l'oppression raciale subie par les francophones de la province la plus populeuse du pays. La 
logique, toute simple, consistera à demander pourquoi les Canadiens français devraient se battre pour libérer les minorités d'Europe ou encore, comme la propagande militaire les y invitait, défendre la civilisation française en défendant la France quand les droits de la minorité française du Canada sont ainsi bafoués ${ }^{19}$.

Le problème du recrutement devint une sérieuse pomme de discorde pour de nombreux Canadiens anglophones. La loyauté à l'égard de l'Empire britannique était pour eux un moyen de démontrer la valeur de la nation canadienne, et les intérêts du Canada étaient inséparables de ceux de la Grande-Bretagne. Pour ce courant de pensée impérialiste et nationaliste, le faible recrutement manifesté au Québec était impardonnable. Des accusations virulentes de déloyauté dans la presse anglophone et le mauvais traitement des recrues de langue française dans l'armée anglophone du Canada ne faisaient qu'empirer les choses. Quand les propositions de conscription furent rejetées au Québec, la presse de langue anglaise vit de plus en plus dans le Canada français un ennemi intérieur. "Un vote pour Laurier, pouvait-on lire dans le Globe de Toronto, est un vote pour le $\mathrm{Kaiser}^{20}$. " Le premier ministre Robert Borden, dans un écrit privé, alla jusqu’à assimiler les «Français » aux " étrangers et (aux) fainéants » qu'il considérait comme déloyaux ${ }^{21}$.

Alarmé par le changement radical observé dans les relations entre Canadiens francophones et anglophones, et désireux de retrouver l'esprit d'unité qui prévalait au début de la guerre, un petit groupe de politiciens et d'hommes d'affaires ontariens entreprit de créer un mouvement de rapprochement. John Milton Godfrey, politicien libéral fédéral et principal recruteur pour le Corps expéditionnaire canadien dans la région de Toronto-Hamilton, était le plus en vue d'entre eux. Lors d'une réunion de la National Service League à Toronto le 16 
juin 1916, Godfrey annonça son idée. Un autre recruteur, le colonel Lorne Mulloy, ancien combattant aveugle de la guerre des Boers, un conservateur, exprima de façon plutôt amusante l'idée initiale du mouvement et sa logique simple et attrayante :

Deux ou trois d'entre nous étions en train de déjeuner [...] à Toronto, et un homme a dit : " J'aimerais emmener 50 Ontariens à Montréal, juste pour leur montrer que les Canadiens français n'ont pas de cornes.» Et quelqu'un a ajouté : « Oui, et si nous pouvions emmener 50 Canadiens français ici pour leur montrer que nous ne nous levons pas la nuit pour aboyer, ce serait une bonne chose. ${ }^{22}$ ”

Comparant leur mouvement à la très célèbre « entente cordiale » entre la France et la Grande-Bretagne, deux vieux ennemis, ils baptisèrent leur mouvement la Ligue de la Bonne Entente (LBE). Le premier objectif de la Ligue devait être alors de retrouver les «bons sentiments » observés pendant les premiers mois de la guerre. Elle n'avait nullement l'intention de régler la question du recrutement. «Il était clairement admis que l'Ontario n'avait pas à influencer de quelque manière le recrutement ou quoi que ce soit relatif à la guerre au Québec $^{23}$.» La LBE avait des objectifs limités - ce devait être la première étape sur la longue route menant à la réconciliation et à l'unité nationale. Cette initiative, poursuit l'histoire officielle, «n'avait pas pour but de régler des controverses, mais d'évaluer les chances de l'établissement de sentiments plus amicaux, sans lesquels le règlement de controverses serait irréalisable ${ }^{24} \gg$.

Godfrey avait tout de même d'autres raisons de vouloir améliorer les relations. Il n'en croyait pas moins que ses intentions concernaient essentiellement l'unité nationale. Impérialiste et méthodiste convaincu ayant la réputation d'être 
anticatholique et anti-français ${ }^{25}$, Godfrey était préoccupé par le faible taux d'enrôlement chez les Québécois francophones. Par ses contacts avec Arthur Hawkes, journaliste bilingue originaire d'Angleterre bien disposé envers les Canadiens français, Godfrey avait adouci ses vues dans une certaine mesure. Godfrey, Hawkes et Mulloy ne préconisèrent pas au départ la conscription. Ils espéraient plutôt qu'en améliorant les relations personnelles parmi l'élite francophone et anglophone, les Canadiens anglais seraient moins susceptibles de faire affront aux francophones, et les Canadiens français deviendraient moins hostiles à l'effort de guerre. Un enrôlement volontaire accru contribuerait à éviter la conscription, le dernier recours potentiellement source de divisions ${ }^{26}$.

Godfrey se mit rapidement à établir les bases de la première conférence de la LBE. Il assuma personnellement la tâche de susciter de l'intérêt dans la haute société ontarienne, tandis que Mulloy et Hawkes se concentraient sur le Québec. Sir Georges Garneau, président de la Commission des champs de bataille nationaux à Québec, lui-même recruteur en temps de guerre, s'avéra une relation précieuse. Avec l'aide de Garneau, Hawkes obtint une invitation de la Chambre de commerce de Sherbrooke pour la visite préliminaire d'une poignée de délégués ontariens. Sherbrooke était considéré comme idéal, car c'était une ville " où les relations entre les deux races sont des plus amicales ${ }^{27} »$. Les délégués furent reçus avec enthousiasme et la réunion s'avéra un succès. Godfrey, Hawkes et Garneau créèrent sur-le-champ un comité mixte de représentants de l'Ontario et du Québec pour organiser des réunions plus importantes dans les deux provinces. À ces réunions participeraient un nombre encore plus grand d'anglophones et de francophones. Le mouvement était officiellement lancé. 
La liste des participants aux conférences de la LBE de 1916 et 1917 était impressionnante. On y retrouvait un éventail de politiciens libéraux et conservateurs des trois ordres de gouvernement, des avocats réputés et des hommes d'affaires prospères, des juges, des recruteurs et des membres des forces armées, des ecclésiastiques et mêmes quelques universitaires. Parmi les participants francophones figuraient le député libéral fédéral nationaliste Paul-Émile Lamarche, Zéphirin Hébert, président de la Chambre de commerce de Montréal, le magnat de la publicité O. S. Perrault, de l'Imperial Tobacco Company, également de Montréal, le maire de Québec, H.-E. Lavigueur, le premier ministre du Québec, Lomer Gouin, et le sénateur libéral Raoul Dandurand. Parmi les participants anglophones, on peut mentionner le maire de Toronto, T. L. Church, le premier ministre de l'Ontario, W. H. Hearst, le révérend Robert Alexander Falconer, président de l'Université de Toronto, le colonel P. A. Guthrie, du Nouveau-Brunswick, un impérialiste pur et dur, S. R. Parsons, vice-président de l'Association des manufacturiers canadiens, et même un fruiticulteur de Beamsville (Ontario), Francis C. Jones $^{28}$.

Pendant quatre jours d'octobre 1916, une cinquantaine de délégués ontariens effectuèrent, avec force repas bien arrosés, une tournée de bienveillance à travers le Québec. En fait, leurs hôtes enthousiastes, au nombre de cent cinquante, firent en sorte qu'ils n'oublient pas leur visite, avec tout le cérémonial de missions diplomatiques de paix et d'amitié. Les hôtes montréalais fournirent pas moins de trente-cinq voitures - quinze de plus que ce qu'ils avaient demandé - aux délégués ontariens nerveux à leur descente du train. Le groupe fut ensuite transporté dans toute la ville, où ils visitèrent toutes sortes d'institutions d'enseignement, d'entreprises et d'industries. Ils conclurent la journée par un banquet au Club Saint- 
Denis, « où les discours des deux côtés couvrirent tout ce qui concerne les relations interraciales dans un esprit de franche cordialité », lit-on dans l'histoire officielle.

Tous étaient d'avis que cette manifestation justifiait à elle seule le pèlerinage. Il s'avéra que la franchise, au lieu de mettre mal à l'aise, produisait immédiatement respect mutuel et désir de connaître plus intimement le point de vue de l'autre. L'hospitalité de Montréal fut sans borne, et la camaraderie des hôtes était un objet d'envie, bien qu'inaccessible au tempérament plus flegmatique des Ontariens ${ }^{29}$.

Les bons sentiments continuèrent à couler comme le vin lors de visites et de banquets semblables à Trois-Rivières, Québec et Sherbrooke. Ils se bourrèrent la panse de mets succulents, soupe aux huittres, saumon de Gaspésie et canard sauvage grillé, et leurs oreilles s'emplirent de propos empreints de bonhomie ${ }^{30}$.

La visite qu'effectuèrent en retour cinquante délégués québécois en Ontario au début de janvier 1917 s'avéra tout aussi cordiale. Les Ontariens offrirent leurs propres banquets le soir à leurs invités québécois et leur firent visiter le jour des universités et des usines de munitions à Toronto et Hamilton. Pour clore le tout, la visite du site ontarien le plus touristique, les chutes du Niagara, s'imposait ${ }^{31}$. Ayant été si bien nourris au Québec, les Ontariens relevèrent le défi culinaire des Québécois en offrant poulet aux truffes, perche King Edward et pêches du Niagara, entre autres délices ${ }^{32}$. Il n'y a pas alors à s'étonner que les discours soient généralement demeurés amicaux et qu'il n'y ait pas eu d'échanges passionnés.

En fait, les organisateurs de la Ligue de la Bonne Entente tentèrent de faire en sorte que les discussions demeurent amicales et exemptes de controverses. Pour eux, l'objectif premier restait qu'un semblant de courtoisie marque les 
relations entre Canadiens anglais et français. À cette fin, ils espéraient éviter, dans la mesure du possible, les questions sources de division, bilinguisme et conscription. Au début, écrit Godfrey, "nous croyions impossible d'aborder des sujets controversés ${ }^{33}$ ». Bien qu'on en parlât en privé et dans la correspondance, la plupart des délégués tinrent compte des souhaits de Godfrey et évitèrent de parler directement de la conscription et de la question générale de la participation aux forces armées. En lieu et place, la plupart des hommes se contentèrent de propos flatteurs et admiratifs et parlèrent d'égalité raciale. Le langage fleuri de Garneau à la conférence de Hamilton en janvier 1917 est tout à fait caractéristique :

Vous êtes venus à nous en octobre dernier croyant qu'en nouant des liens plus étroits avec nous, vous vous rendriez compte que le sang qui coule dans nos veines est aussi riche et chaud que le vôtre, et que nous ne pouvions que trouver les idéaux communs et les accords que nous cherchions. Nous sommes venus à vous ayant, absolument et fermement, confiance dans l'authentique chaleur profonde, saine et large d'esprit de la population ontarienne. Avons-nous été déçus, messieurs ? Je n'ai qu'à poser cette question aux hommes de notre délégation de la province de Québec pour obtenir sans délai une réponse. (des voix : $\mathrm{Non}^{34}$.)

Quand le sujet de la guerre venait sur le tapis, les hommes parlaient du courage des soldats au front, et du glorieux héritage militaire des peuples français et britannique. Comme on pouvait s'y attendre, cette thétorique revenait dans les propos des militaires, tant francophones qu'anglophones. En fait, on y avait particulièrement recours pour dissiper les doutes quant à la loyauté du Québec à l'égard de la cause alliée. Les références ne manquaient pas à la bravoure du $22^{e}$ Bataillon (canadien-français), qui venait de remporter une victoire à Courcelette, sur la Somme, en septembre $1916^{35}$. 
Chose intéressante, des responsables non militaires tenaient également de tels propos. "On trouve souvent des noms du Québec sur les listes des pertes de la guerre, déclarait le premier ministre du Québec, Lomer Gouin, ce qui montre que la fidélité est la même, et aussi forte, chez les Canadiens français d'aujourd'hui ${ }^{36}$. » Gouin cherchait peut-être ainsi à réfuter discrètement l'argument en faveur de la conscription, affirmant que le Québec faisait déjà sa part, et plus. Le premier ministre ontarien W. H. Hearst, pour sa part, profita de l'occasion pour dénoncer la politique partisane et exprimer sa position sur la question de la guetre, se montrant indirectement en faveur de la conscription.

À ce moment de l'histoire du Canada, nous ne pouvons penser qu'à la guerre $[\ldots]$ rien d'autre dans le monde ne compte $[\ldots]$ Cela exige non seulement ce qu'il y a de mieux dans un Canada uni, mais ce qu'il y a de mieux dans l'Empire uni, partout où flotte le drapeau britannique dans le vaste monde. (applaudissements $^{37}$ )

Pour Hearst, un front uni exigeait le soutien inconditionnel de tous les détenteurs du pouvoir, y compris pour la conscription si elle devait être décrétée. Il faut noter que, bien que le premier ministre fût prêt à faire des allusions à la conscription, il évita toute mention des droits des francophones.

Donc, si on lit entre les lignes des discours cordiaux, le mouvement semble avoir eu des significations différentes pour les uns et les autres. Les participants étaient après tout fort divers. Sans parler de la séparation entre Français et Britanniques, entre Québec et Ontario, la diversité des intérêts professionnels signifiait que les participants au mouvement avaient inévitablement des points de vue différents. Bref, chaque homme apportait avec lui dans les salles de banquet son propre ordre du jour. Beaucoup des hommes d'affaires et des 
industriels présents aux réunions de la Ligue de la Bonne Entente, par exemple, s'intéressaient moins aux questions politiques et davantage aux problèmes économiques. Des hommes tels que Zéphirin Hébert ou S. R. Parsons, en arrivant à la conférence, pouvaient sans l'ombre d'un doute être mis au rang de ceux qui craignaient que l'animosité que la guerre avait engendrée entre Québécois et Ontariens encourage des boycotts interprovinciaux. En conséquence, les deux premiers ministres provinciaux et les différents maires présents aux conférences parlèrent de la nécessité d'une solidarité économique. La Ville de Trois-Rivières distribua même une brochure, "To Our Guests From Ontario », qui les invitait ouvertement à investir dans cette partie du pays :

Vous êtes venus dans l'intérêt des grandes institutions professionnelles, industrielles et commerciales pour lesquelles votre province honnête et industrieuse est célèbre à juste titre, $[\ldots]$ pour voir de vos yeux sensibles et pratiques les nombreux avantages que nous prétendons offrir, pour étudier d'un coup d'œil nos ressources naturelles principale ${ }^{38}[\ldots]$

Pour certains des bonne-ententistes les moins sincères, le mouvement était moins un exercice de rapprochement qu'une opération commerciale.

Certains des recruteurs les plus militants tels que les colonels P. A. Guthrie et R. W. Leonard, le président d'une société minière, s'étaient joints au mouvement simplement parce qu'ils espéraient que ce dernier « influencerait favorablement l'opinion au Québec en faveur de l'enrôlement ou de la conscription ${ }^{39} \%$. Godfrey ne tarda pas à mettre en garde contre une telle présomption. "Je ne préconise pas une campagne dans la province d'Ontario dans le but d'obliger le Québec à accepter l'enrôlement et la contrainte, dit-il à un recruteur inquiet du Québec. Notre position en Ontario de- 
vrait être que notre relation doit être amicale, et que ce n'est pas le moment pour cette province de forcer qui que ce soit à adopter une opinion ${ }^{40}$. " Hawkes donnait le même conseil : "Nous devons éviter de laisser entendre que nous essayons d'imposer quelque chose au Québec ${ }^{41}$. » Au fond, Godfrey, Mulloy, Hawkes et Garneau espéraient effectivement que l'esprit d'amitié engendré par le mouvement adoucisse indirectement certaines prises de position contre l'enrôlement au Québec. Mais c'était pour le moment une priorité secondaire elle demeurait subordonnée à la nécessité de changer les attitudes en Ontario et de mettre un terme aux attaques insultantes contre la loyauté des Canadiens français ${ }^{42}$.

Sans cesse présents dans l'esprit des participants, les sujets de controverse ne pouvaient se cantonner aux remarques indirectes. Godfrey fit ainsi observer : "Cette volonté [d'éviter les sujets controversés] a été dissipée par la suite par le discours remarquablement franc et honnête prononcé par Paul Lamarche au banquet de Montréal ${ }^{43}$. " Lamarche, un nationaliste, exposa ses vues très clairement lors de la réunion inaugurale de Montréal, le 9 octobre 1916 : «Nous avons, ici, la conscription non de fait, mais de force, la conscription du pauvre forcé de s'enrégimenter par la main pesante du riche. » Sur les droits de la langue française : « la chose la plus pénible pour un Canadien, [est d'être] privé du privilège de parler français $^{44}$. " À Hamilton en janvier 1917, le sénateur libéral Raoul Dandurand profita également de l'occasion pour s'exprimer longuement en faveur des droits des francophones et mit en garde en général contre "les préjugés qui existent à travers votre province contre les Français ${ }^{45} »$. Pour leur part, quelques anglophones en profitèrent pour défendre les politiques linguistiques plutôt intransigeantes de l'Ontario. La plupart des anglophones et des francophones, toutefois, évitèrent soigneusement le sujet des droits de la langue française, se 
contentant de vagues engagements en faveur de la tolérance et de l'amitié.

Fait révélateur, quelques anglophones se montrèrent bien disposés envers le point de vue canadien-français. C'étaient généralement des personnes qui avaient vécu au Québec, ou encore qui connaissaient bien la langue française et l'histoire du Canada français. Par exemple, John Squair, professeur de français à l'Université de Toronto, se montra très favorable aux principes de réconciliation et de compréhension mutuelle:

On dit que vous avez déclaré ce matin que vous désiriez des mesures de conciliation relativement aux affaires du Bas-Canada. [...] Vos paroles sont les plus sensées que j’ai entendues depuis longtemps. Je crois que nous serons confrontés à de graves difficultés si nous ne pouvons vivre en paix avec nos concitoyens canadiens-français ${ }^{46}$.

Squair, qui avait étudié «le dialecte franco-canadien», s'intéressa tout de suite au mouvement, prenant part «à presque toutes ses activités ». Il avait travaillé avec l'Ontario Education Association, et n'avait que des louanges pour le système d'éducation québécois, qui avait constamment fait l'objet de critiques de la part de ceux qui tentaient de légitimer la suppression par l'Ontario de l'enseignement en langue française ${ }^{47}$. Un collègue de Squair à l'Université de Toronto, le révérend George McKinnon Wrong, un anglican, participa aussi à la Ligue de la Bonne Entente. Professeur d'histoire et d'ethnologie, Wrong encourageait l'étude de l'histoire du Canada. L'histoire de la Nouvelle-France figurait parmi ses intérêts relatifs au passé du Canada ${ }^{48}$. Pour ces hommes, le mouvement de la bonne entente constituait une occasion d'influencer favorablement l'opinion en Ontario. 
Arthur Hawkes était également de cet avis. Bien que né en Angleterre, l'éloquent Hawkes, qui était bilingue, avait vécu plusieurs années tant au Québec qu'en Ontario, où il avait écrit et donné des conférences ${ }^{49}$. Son espoir premier que le mouvement serve à restaurer l'unité nationale - d'abord et avant tout en modérant l'opinion en Ontario - est tout à fait évident dans une lettre qu'il écrivit à Laurier à la suite de la proposition initiale de Godfrey concernant la «bonne entente » :

J'ai assisté, un peu à contrecœur, à une réunion de la National Service League qui m'a plutôt surpris. Une discussion des relations entre le Québec et l'Ontario a révélé une volonté très agréable de coopération cordiale entre les deux, par des conférences où l'on tiendrait à apprécier et à accepter le point de vue du Québec. Ce qui a eu lieu a confirmé, de façon très agréable, ce que je vous ai souvent affirmé, que le point de vue de l'Ontario peut être modifié de manière positive si l'on considère la situation de façon plus générale. Je ne suis pas membre de la ligue, mais la réunion a témoigné d'une volonté d'accueillir toute coopération. $[\ldots]$ cette suggestion me séduit énormément ${ }^{50}$.

John Boyd, conseiller bilingue à la Ville de Montréal, fut plus explicite : aux conférences en Ontario, il demanda ouvertement pourquoi, compte tenu du traitement généreux de la minorité anglophone au Québec, les francophones de l'Ontario ne pouvaient être traités aussi justement. Un Godfrey embarrassé dut mettre un terme au discours de Boyd « faute de temps ${ }^{51} »$. Finalement, la Ligue de la Bonne Entente de 1916-1917 réussit dans l'ensemble à éviter les controverses, car les participants succombèrent plus ou moins à la bonhomie générale que Godfrey avait espérée. 


\section{L'après-guerre et l'évolution du mouvement de la bonne entente}

En février 1917, Godfrey, Mulloy et Hawkes firent porter leurs efforts sur la création de la convention Win-the-War « dans le but de suggérer et de promouvoir des mesures dynamiques pour hâter l'issue positive de la guerre ${ }^{52}$ ». Les conséquences de l'échec imprévu sur la Somme en 1916 nous tombaient tout juste dessus - dont 24029 pertes pour le Corps canadien $^{53}$ - et avaient confronté les recruteurs à une nouvelle réalité. Les recruteurs plus modérés avaient jusque-là espéré éviter la conscription, mais elle semblait maintenant s'imposer. La convention bipartisane eut lieu en mai 1917, réunissant des délégués de tout le pays pour appuyer les deux politiques, gouvernement de coalition et conscription. Dans ce que l'historien Brian Cameron a appelé « un mauvais jugement sans pitié qui eut pour effet d'annuler en grande partie, sinon totalement, l'esprit de bienveillance qu'avait permis la [Ligue de la Bonne Entente] », les organisateurs de Win-theWar "trouvèrent l'idée d'obtenir l'appui des "bonneententistes" du Québec en décrivant la réunion comme une "convention de l'unité nationale [...] pour discuter des problèmes nationaux et économiques issus de la guerre" $" 54$.

Les bonne-ententistes canadiens-français participant à Win-the-War en partant du principe qu'il s'agirait d'une convention sur l'unité nationale furent cruellement déçus quand ils découvrirent son véritable objectif. Trois jours avant la convention, le premier ministre Robert Borden annonça son projet de conscription. La plupart des délégués à Win-theWar appuyaient ce projet, ainsi que le gouvernement de coalition. Les questions relatives à l'unité nationale passèrent au second $\operatorname{plan}^{55}$. "Nous avons été si cruellement trompés par l'Ontario, écrivit un bonne-ententiste canadien-français désillusionné, que ceux d'entre nous qui avaient cru en la sincé- 
rité et en l'honnêteté du mouvement de la bonne entente furent humiliés par le fait qu'on nous avait eus comme une bande d'écoliers ${ }^{56} \%$. Moins d'un an après, l'élection du gouvernement de coalition de Borden créa une scission parlementaire entre groupes linguistiques, et la conscription fut imposée, ce qui indigna grandement les francophones du Québec. Le pays était plus divisé que jamais, et pour le moment l'idéal de la bonne entente semblait mort.

Il serait trompeur de laisser entendre, cependant, que Win-the-War était simplement une continuation du mouvement de la bonne entente. Certains des bonne-ententistes les plus modérés, dont des anglophones, continuèrent de promouvoir la cause de la compréhension mutuelle. Pour sa part, John Boyd s'affairait à distribuer des publications à des journaux et à des politiciens à travers le pays. Dans une lettre ouverte à La Presse en février 1918, Boyd s'excusa au nom des Canadiens anglophones :

Ce serait une grande erreur que les Canadiens français croient que tous les Canadiens de langue anglaise leur sont hostiles ; au contraire, beaucoup de Canadiens anglophones ont vigoureusement protesté contre les attaques injustes à l'encontre des Canadiens français et ont montré qu'ils sont en faveur d'une justice complète pour leurs compatriotes d'origine française. [...] Les attaques contre les Canadiens français émanent d'une minorité relativement petite, et non de la masse des Canadiens anglophones, qui sont de bonnes personnes ayant le souci de l'équité.

Le défi, poursuivait-il, était d'éduquer les Canadiens anglophones :

Le principal obstacle à surmonter est le manque de connaissances chez un grand nombre de Canadiens de langue anglaise relativement à la situation réelle des $\mathrm{Ca}$ nadiens français. Ce qui est donc le plus essentiel, c'est 
que la position réelle des Canadiens français devrait être présentée clairement, équitablement et entièrement aux Canadiens anglophones à travers le Dominion, et c'est à ce travail que je consacre actuellement mon humble énergie dans l'intérêt de la concorde raciale et de l'unité nationale ${ }^{57}$.

Hawkes, qui avait depuis le début vu dans la LBE un moyen d'influencer l'opinion publique en Ontario, avait pour sa part été cruellement déçu quand le mouvement avait été lié par erreur à Win-the-War ${ }^{58}$. Même Godfrey et Mulloy allaient reprendre leurs activités en faveur de la "bonne entente " une fois la guerre terminée.

Loin d'être abandonné, le mouvement fut relancé officiellement à l'été 1918. Il s'agissait cette fois de la «Better Understanding Association » (BUA), qui fut surnommée dans la presse francophone la "Meilleure Entente ${ }^{59}$ ». On y trouvait des membres nouveaux et anciens, et elle devait être une association permanente ayant son siège à Ottawa. La nouvelle association avait comme fer de lance William Charles Mikel. Cet avocat prospère avait grandi à Belleville (Ontario), ville qu'il décrivait comme un carrefour entre sa province et le Québec ${ }^{60}$. Mikel était aussi le président de l'Association fraternelle canadienne (AFC). Formée dans les années 1890, l'AFC était une organisation chapeautant divers clubs et organisations sociales tels que l'Ancient Order of United Workmen, l'Alliance nationale, la Loyal Orange Lodge et l'Union Saint-Joseph du Canada. Parmi les dirigeants de l'AFC, on trouvait tant des francophones que des anglophones. D'après Mikel, elle eut une influence non négligeable à travers le pays, car les groupes qui lui étaient associés comptaient quatre cent mille membres, francophones et anglophones, protestants et catholiques ${ }^{61}$. Cela le mettait dans une position unique pour avoir des contacts avec une liste diverse 
de participants. Mikel organisa la réunion initiale de deux jours en juillet 1918, avec vingt-huit notables et représentants des diverses organisations affiliées à l'AFC du Québec et de l'Ontario. Elle devait être suivie d'autres réunions, en novembre 1918 et en septembre 1920.

Dans l'ensemble, les participants à la BUA étaient des politiciens, des professionnels et des pédagogues. Chez les anglophones, notons E. O. Platt, le maire de Belleville, le $\mathrm{D}^{\mathrm{r}}$ J.W. Edwards, orangiste et député conservateur fédéral de Cataraqui, le colonel William Nesbitt Ponton, avocat de Belleville des plus favorables aux préoccupations des Canadiens français, et James B. McKillop, procureur de la Couronne de London (Ontario). Mulloy et Godfrey prirent également part aux nouvelles délibérations. Parmi les participants francophones figuraient l'ancien maire de Montréal et membre de l'Alliance nationale, L. A. Lavallée, J. A. A. Brodeur, un conseiller municipal de Montréal, et Rodolphe Bédard, président de la Société des artisans canadiens-francais. Fait révélateur, l'Association comportait aussi une représentation francoontarienne, dont O. J. Rochon, membre de l'Union Saint-Joseph du Canada, Napoléon Champagne, conservateur membre de la Société des artisans canadiens-français, et à l'époque maire par intérim d'Ottawa, et Aurélien Bélanger, qui avait été inspecteur d'école à Ottawa, farouche défenseur de la qualité de l'enseignement dans les écoles ontariennes de langue française ${ }^{62}$.

Les objectifs de la BUA étaient proches de ceux de son prédécesseur. Mikel était mû par l'inquiétude sincère que, à la lumière de récents événements, le pays soit " déchiré » si une «meilleure compréhension entre Canadiens francophones et anglophones " ne se manifestait pas. Il avait présenté cette idée devant l'AFC à la fin d'avril 1918. Au début du mois, la crise de la conscription était devenue critique quand quatre 
civils furent tués et dix soldats blessés au cours d'une émeute à Québec. «Le moment présent est tout à fait favorable psychologiquement à cette réunion ", déclara-t-il aux membres de l'AFC ${ }^{63}$. Les propos liminaires de Mikel à la réunion de la BUA allaient dans le même sens :

Une des difficultés de la situation est le fait que les gens de la province de Québec et du reste du Dominion ont très peu de contacts les uns avec les autres et se connaissent très peu. L'absence de relations entre les populations du Nord et du Sud est considérée comme une des causes principales de la guerre de Sécession. [...] Que les sociétés fraternelles, par leurs sections, procurent cette connaissance aux gens ${ }^{64}$.

Certains délégués qui venaient d'adhérer au mouvement de la bonne entente affichaient un optimisme caractéristique. Le maire Platt, de Belleville, parla d'une « réunion qui fera date ${ }^{65}$ ». Un autre délégué alla plus loin: "Je ne pense pas qu'en ce moment dans le Dominion du Canada il y ait de réunion plus importante que celle-ci, ni de réunion pouvant produire des résultats aussi importants. ${ }^{66}$ "

Certains des nouveaux venus partageaient l'enthousiasme naif de leurs prédécesseurs, mais ceux qui connaissaient bien la vieille LBE tenaient à ne pas répéter ses erreurs. En fait, le surnom de "meilleure entente » voulait peut-être distinguer la BUA de son prédécesseur. À cette fin, les réunions étaient à échelle beaucoup plus petite et l'apparat était moindre que dans le cas de la ligue originelle. De plus, Mikel voulait aller au-delà de ce qui intéressait simplement l'élite commerciale et politique, et hors de l'Ontario et du Québec. Il voulait atteindre le grand public et toutes les classes en général, avec des programmes qui, par exemple, créeraient des possibilités d'échange entre jeunes du Québec et des autres provinces du Canada. En quelques années, prétendait-il, ces 
manifestations "suffirai[en]t à modifier la mentalité actuelle $^{67}$ ». La tonalité des réunions du nouveau mouvement de la bonne entente fut différente de celle des années précédentes, car Mikel encourageait les participants à dire ce qu'ils pensaient - à s'exprimer franchement sur les sujets brûlants. Mikel distinguait entre le nouveau et l'ancien mouvement :

On a dit de la Ligue de la Bonne Entente qu'ils organisaient de gros banquets et qu'ils se donnaient généralement du bon temps, mais l'opinion des gens était que le contenu des délibérations n'était pas sérieux. Considérons cela comme une proposition sérieuse, et qu'il ne faut pas confondre les délibérations avec des fêtes, des divertissements et de bons moments ; faisons quelque chose d'utile pour le pays ${ }^{68}$.

Nombre de participants étaient d'accord. «Nous ne pouvons pas nous contenter de nous réunir ici pour nous congratuler les uns les autres, porter des queues de pies et assister à des dîners chic et penser que nous allons transformer le monde parce que nous sommes gavés d'huîtres, proclama l'un d'eux. Nous devons nous appuyer sur des faits solides et nous devons nous montrer résolus en tant que Canadiens ${ }^{69}$. $\gg$ Ils allaient créer une tribune constructive.

Le plus éminent des bonne-ententistes de la première heure présent à cette occasion était le colonel Mulloy. Ayant retenu les leçons de la première initiative de bonne entente, il faisait preuve d'un optimisme prudent: «Nous avons laissé les deux grandes races qui forment la souche de la nation déclencher une bagarre familiale extrêmement regrettable et inconvenante. " Il poursuivait en attaquant la presse et les politiciens exaltés et opportunistes - osant une allusion risquée aux extrémistes orangistes - qu'il accusait d'exagérer l'hostilité existante et de l'attiser. Il sympathisait ouvertement avec ceux des Canadiens français qui étaient persuadés d'être in- 
justement opprimés : «Si vous réussissez à persuader un $\mathrm{Ca}$ nadien français qu'il est persécuté, victime de prussianisation, il ne serait pas un homme s'il ne se battait pas pour protéger ce qui lui appartient ${ }^{70}$. " Napoléon Champagne était d'avis comme Mulloy que « l'ensemble des francophones et l'ensemble des anglophones ne peuvent être jugés par une poignée de têtes brûlées ${ }^{71}$ ».

Étant donné la franchise de ton de la conférence, la question des écoles de l'Ontario et des droits des Canadiens français furent inévitablement au cœur des débats et donnèrent lieu à de vives discussions. Le $\mathrm{D}^{\mathrm{r}} \mathrm{J}$.W. Edwards se lança dans une défense assez longue du Règlement XVII. Le Canada, déclarait-il, est un pays anglophone et doit le demeurer s'il veut prospérer. En tant que tel, la langue française n'a aucune reconnaissance constitutionnelle en Ontario ni nulle part ailleurs au Canada - sauf, peut-être, au Québec. Le français ne doit pas non plus jouir d'une plus grande reconnaissance constitutionnelle si on ne veut pas assister à la balkanisation du pays. Il affirmait que dans certaines parties de l'Ontario les enfants anglophones protestants n'avaient guère d'autre choix que d'aller dans des écoles privées francophones proches où le niveau d'instruction était apparemment inférieur ${ }^{72}$. Ces allégations furent âprement contestées par les délégués franco-ontariens, Champagne et Aurélien Bélanger.

Chose intéressante, ce n'est pas d'un francophone, mais du colonel Mulloy que vint l'opposition la plus forte et la réfutation la plus éloquente. Fils d'orangiste et ancien combattant de la guerre des Boers, Mulloy demeura un impérialiste et un conservateur. Mais l'expérience de la LBE avait profondément modifié la façon de penser de Mulloy et d'autres. «Le résultat, rappelait-il, fut de débarrasser pour toujours l'esprit de ces cinquante hommes, d'une bonne partie, sinon de la totalité, des préjugés et de la méfiance qui étaient les leurs ${ }^{73}$.» 
La défense énergique des droits du français de Mulloy mérite d'être citée en entier, car elle montre à quel point sa vision du Canada avait évolué, et témoigne de son sens des responsabilités :

Certes, il est vrai que la langue française n'est pas reconnue par la loi en Ontario. Il est vrai que l'Acte de Québec et que d'autres lois [...] ne mentionnaient pas la langue française. Mais c'était sous-entendu. C'est pourquoi on ne l'a pas mentionné. Et nous, les anglophones du Canada, si nous voulons nous en tenir à l'aspect légal de la chose, nous renoncerons à notre sécurité morale. Je ne prends pas fait et cause pour le Canada français. Je parle simplement en mon nom, en tant que citoyen de ce jeune Dominion, et je donne mon sentiment, le sentiment que nous ne devons pas renoncer à notre sécurité morale et que nous ne le ferons pas. Le Canadien français $[. .$.$] est ici pour rester, et vous ne$ pouvez pas légalement le chasser de l'Ontario ou du Canada, pas plus que vous ne pouvez bannir par la loi la langue qu'il parle. Il est ici pour rester et nos relations doivent être régies par l'équité et la justice si nous voulons un jour devenir une nation.

Le colonel, allant plus loin, rejetait l'ancien nationalisme ethnique des Canadiens anglais en faveur d'un nationalisme civique à la manière de George-Étienne Cartier :

J'appartiens à ce groupe de Canadiens qui ne sont pas moins fiers, mais plus fiers de leur citoyenneté canadienne parce que le Canada est formé des valeureux descendants de l'ancienne et chevaleresque France. Vous, hommes du Canada français, possédez ce que nous n'avons pas ; nous avons ce que vous ne possédez pas et nos cultures respectives, en interagissant entre elles, donneront naissance à une grande nation ${ }^{74}$. 
Le débat prit rapidement un tour personnel lorsque Mulloy mit en doute la valeur des politiciens de la Colline du Parlement et de Queen's Park qui s'opposaient si farouchement aux droits des francophones. «Ils sont une bonne représentation, tant moralement qu'intellectuellement, de la classe de gens qui les a envoyés à Ottawa et à Toronto, rétorqua Edwards. C'est un fait, que ça vous fasse plaisir ou pas. » Mulloy répliqua vivement:

Mulloy: Je suis tout à fait d'accord avec vous pour dire que le pays a le gouvernement qu'il mérite.

Edwards : Je vais encore plus loin, il a un bien meilleur gouvernement que ce qu'il mérite.

Mulloy : Vous savez, pour pouvoir se percher haut, il faut pouvoir voler haut. Néanmoins, il ne faut pas voler au-delà du perchoir, mais nous voulons de meilleures politiques et une meilleure citoyenneté au Canada, et nous allons avoir les deux ${ }^{75}$.

Enhardi par la vigoureuse défense des droits de la langue française par le colonel, Bélanger se lança dans la bataille. Les Canadiens français d'Ontario, affirma-t-il, «sont, si l'on peut dire, le chaînon manquant, dans l'évolution [...] qui doit se produire si une nationalité canadienne digne de ce nom doit un jour émerger. » Il ajouta que le traitement réservé à la minorité franco-ontarienne était considéré au Québec comme " pratiquement un premier pas vers l'invasion de la province de Québec et l'utilisation des mêmes méthodes ou de méthodes similaires pour l'angliciser totalement ${ }^{76} \%$. Il évoqua lui aussi un nationalisme civique exigeant la participation active des deux sociétés de l'État canadien: «Nous voulons rivaliser avec vous pour faire du Canada le meilleur pays du monde, nous voulons, par notre patriotisme, faire, si possible, encore plus que vous ne faites, non seulement pour le Canada dans son ensemble, mais en particulier pour l'On- 
tario. » Il conclut son allocution en faisant appel, tout comme Mulloy, à la conscience morale de ses collègues anglophones : "Nous ne sommes nullement démoralisés, et nous n'avons aucunement l'intention d'user de représailles, parce que nous avons une confiance solide et constante dans le fait que c'est finalement le fair-play naturel des Anglais de cette province qui prévaudra. (Bravo!) ${ }^{77}$. » Malgré ce plaidoyer, la conférence s'acheva sans que les participants en soient arrivés à un consensus sur la question de la langue.

C'est ce manque de consensus même qui incitait les chefs de la BUA à organiser une conférence après l'autre. Ils étaient fermement persuadés que le premier pas le plus judicieux à accomplir pour traiter les doléances tant des Canadiens anglais que des Canadiens français était de les formuler clairement et avec franchise. Lors de la deuxième conférence, qui eut lieu au Château Laurier à Ottawa, le 28 novembre 1918, John Godfrey décrivit les réussites et les échecs du premier projet de bonne entente. Les participants s'entendirent pour mettre sur pied un sous-comité composé de Godfrey, Edwards, Mikel, Bélanger, Champagne et Rodolphe Bédard, président de la Société des artisans canadiens-français, "pour dresser une liste provisoire des différences entre les Français et les Anglais au Canada, dans le but d'essayer, si possible, de réconcilier leurs différences ${ }^{78}$ ». Parmi les « différences » énumérées, figurait, encore une fois, en tête de liste la question des droits des francophones en Ontario, de plus en plus considérée par les bonne-ententistes des deux communautés linguistiques comme la source principale de toutes les rancœurs ${ }^{79}$.

Lors de la troisième réunion de la BUA, qui se tint encore une fois au Château Laurier, en septembre 1920, les délégués s'entendirent pour créer une organisation permanente ${ }^{80}$. La BUA réussit à obtenir divers appuis importants. Grâce à l'influence de Mikel, l'Association du Barreau canadien adopta 
au cours de sa réunion annuelle de 1921 une résolution « demandant avec insistance aux membres de la profession juridique d'user de leur influence pour favoriser une meilleure entente entre les Canadiens de langues française et anglaise ${ }^{81}$ ». Les réunions et les activités du mouvement attiraient maintenant un «nombre d'éminents hommes d'État canadiens» de plus en plus élevée $e^{82}$. En 1921, Mikel reçut des lettres de soutien de tous les premiers ministres provinciaux ainsi que du premier ministre fédéral Arthur Meighen et du chef de l'opposition officielle de l'époque, William Lyon Mackenzie King $^{83}$.

D'autres sympathisants continuèrent d'exercer des pressions en faveur de l'idéal de la bonne entente jusque vers la fin des années 1920, avec l'intention de plus en plus affirmée d'obtenir l'abrogation du Règlement XVII. Le plus éminent de cette nouvelle génération de bonne-ententistes était le sénateur libéral franco-ontarien Napoléon Belcourt. Avocat réputé et membre du corps enseignant de l'Université d'Ottawa ${ }^{84}$, Belcourt fut président de l'Association canadiennefrançaise d'éducation d'Ontario (ACFÉO) de 1910 à 1912, et de nouveau de 1919 à 1932. Considéré par plusieurs comme le leader des francophones de l'Ontario, Belcourt poursuivit inlassablement son action jusqu'à l'abolition officielle du Règlement en 1927. Il rédigea et diffusa des documents pédagogiques, écrivit des articles de journaux, correspondit avec des figures politiques éminentes et prononça des discours, tant devant des auditoires hostiles que des publics réceptifs ${ }^{85}$. En 1924, le nationaliste ultramontain Lionel Groulx décerna à Belcourt le « Grand Prix d'Action française » en récompense de ses efforts.

Malgré son association avec Groulx, Belcourt était un homme pragmatique ${ }^{86}$. Sous sa direction, l'ACFÉO, à partir de 1921, travailla principalement à tenter de convaincre «la 
majorité protestante de la province de la justice de nos revendications ». Pendant des années, le gouvernement fédéral s'était abstenu d'intervenir sur la question des écoles et Belcourt savait que pour que les choses changent, il fallait atteindre ceux qui détenaient le pouvoir au niveau provincial. Pour changer l'opinion publique, et, plus précisément, l'opinion du gouvernement de l'Ontario, la stratégie de Belcourt était de s'allier avec des Anglo-Ontariens.protestants influents du milieu politique et universitaire ${ }^{87}$.

Le résultat, en 1921, fut la création de la Unity League of Ontario (ULO) - dont le but principal était l'abrogation du Règlement XVII. En 1923, la ligue comptait quelque 150 membres. Tous, sauf un, étaient des anglophones d'une confession autre que catholique. Elle était composée de professeurs d'université, de politiciens, d'avocats, de médecins, de journalistes, d'hommes d'affaires et de « représentants de toutes les sectes religieuses et de tous les partis politiques, progressistes, libéraux et conservateurs ». Selon les membres de la direction de l'ACFÉO, ces personnes avaient une influence positive, tant dans les journaux que dans le public, ainsi qu'au sein du cabinet provincial.

Au moyen de leur influence, de leurs écrits mis au service de notre cause, ces hommes ont créé dans l'Ontario en faveur du français, un mouvement qui se grossit de jour en jour. Depuis deux ans, quelques-uns d'entre eux font littéralement le siège de l'opinion publique anglaise de la province pour lui montrer sous un vrai jour notre cause scolaire et s'efforcent d'obtenir du gouvernement des modifications opportunes ${ }^{88}$.

Fait révélateur, l'ULO comptait parmi ses membres un certain nombre de personnes qui avaient œuvré au sein de la BUA et de la LBE, notamment les professeurs John Squair et E. W. Wrong. Bien que les moins sincères des bonne- 
ententistes se fussent écartés depuis longtemps de l'esprit du mouvement, ce n'était manifestement pas le cas de ces hommes-là - les succès et les échecs des projets de bonne entente antérieurs s'étant avérés formateurs. À Squair et Wrong s'ajoutaient un certain nombre d'autres éminents universitaires, dont C. B. Sissons et O. D. Skelton, également de l'Université de Toronto. L'ULO œuvrait en collaboration constante avec l'ACFÉO. «[C]es amis de notre cause, pouvait-on lire dans un rapport interne de l'ACFÉO de 1923, sont en relations constantes avec nos représentants à Ottawa et ils ne prennent aucune initiative nouvelle sans l'avoir discutée avec [n]otre comité exécutif ${ }^{89}$. "

Tandis que l'ACFÉO et l'ULO travaillaient d'arrachepied à l'abrogation du Règlement XVII, l'ancienne LBE n'était pas tout à fait disparue de la scène - le sénateur Belcourt en personne prit part à ses activités courantes. En 1923 et 1925, respectivement, la LBE organisa de grands banquets semblables à ceux de 1916 et 1917, d'abord à Toronto, puis à Montréal et à Québec. S'il faut en croire La Patrie, le banquet de Montréal, à lui seul, attira cinq cent participants. Il réunit des personnes aussi influentes que Vincent Massey (un futur gouverneur général du Canada et partisan du bilinguisme), les maires de Montréal et de Toronto, Charles Duquette et Thomas Foster, ainsi que les premiers ministres du Québec et de l'Ontario, Alexandre Taschereau et Howard Ferguson ${ }^{90}$ Les banquets étaient ostensiblement présentés comme des manifestations commerciales, ce qui incita L'Action catholique à les qualifier de " fiascos ${ }^{91}$ ». Ils permirent néanmoins de faire entendre aux défenseurs du Règlement XVII, comme au conservateur pur et dur qu'était Ferguson, des arguments en faveur des droits du français. Le lieutenant-gouverneur de l'Ontario, Henry Cockshutt, profita notamment de l'occasion pour se déclarer en faveur des droits du français et affirmer la néces- 
sité pour les francophones et les anglophones de se réconcilier $^{92}$.

Fait intéressant, un des principaux alliés de Belcourt au sein du groupe de pression en faveur de l'abolition du Règlement XVII était le fondateur même de la LBE, John Godfrey. L'historien Brian Cameron a prétendu que "manifestement, la "bonne entente" n'avait pas amélioré la compréhension de Godfrey ${ }^{93}$ ». Mais un examen plus approfondi des activités bonne-ententistes de Godfrey après la guerre au sein de l'ULO dément cette affirmation. Godfrey était devenu membre de l'ULO dès 1922. "Ceux d'entre nous qui ont eu des liens avec la «bonne entente» originelle, écrivait-il en 1924, en sont venus à la conclusion qu'il est impossible d'instaurer une bonne entente permanente à moins que quelque chose ne soit fait pour porter remède aux motifs de doléance que les francophones sont convaincus d'avoir à cause du Règlement $\mathrm{XVII}^{94}$. » Reconnaissant qu'à la racine du problème se trouvaient certaines mentalités prévalant en Ontario, Godfrey s'était joint à l'ULO «pour éduquer l'opinion publique et, en même temps, pour empêcher que le problème ne devienne un problème politique... Si l'incendie des préjugés raciaux devait jamais se rallumer, il serait impossible d'en arriver à une entente équitable ${ }^{95} \%$. De plus, il n'était que trop conscient que les crises intérieures provoquées par la Grande Guerre avaient failli détruire le pays. L'ULO offrait une possibilité de corriger les erreurs du passé.

Godfrey s'efforçait principalement de convaincre sir Arthur Meighen, alors chef de l'opposition à la Chambre des Communes et ami intime du premier ministre de l'Ontario Howard Ferguson. «Il est certain que les francophones considèrent le Règlement comme une insulte à leur nationalité ", écrivait-il à Meighen en 1924. «Le règlement est devenu une obsession nationale et ils exigent sa disparition en témoignage 
de bonne foi. » Le temps était venu, affirmait-il, d'éliminer cette loi exécrée : «Si on leur en donne les moyens nécessaires, les Canadiens français de l'Ontario sont tout à fait d'accord pour que leurs enfants apprennent l'anglais. Cela étant, pourquoi ne devraient-ils pas disposer de tous les moyens possibles pour se perfectionner dans leur propre langue ${ }^{96}$ ?" Godfrey joignit un exemplaire de The Status of the French Language in Canada de Belcourt à son message à Meighen, ainsi qu'une invitation à assister à la " $3^{\text {rd }}$ Historical Lecture " de la Ligue de la Bonne Entente au Château Laurier - dont l'hôte n'était autre qu'Arthur Hawkes ${ }^{97}$. Hawkes avait fait le siège du Premier ministre Mackenzie King, lequel avait fini par être d'opinion que c'était un homme ayant «des idées très justes $^{98} \gg$. Pour sa part, Meighen refusa de s'engager. Mais dans des discours ultérieurs il se montra de plus en plus disposé à ce que soit réglée la difficile situation des Franco-Ontariens, et dans sa correspondance avec Ferguson il donnait l'impression d'encourager le premier ministre à prendre une décision favorable à leur endroit ${ }^{99}$. Entre-temps, Godfrey avait commencé à écrire directement à Ferguson. Avec Belcourt, il élabora un plan pour jouer sur l'intérêt de Ferguson pour la pédagogie en insistant sur le fait que les enfants unilingues francophones pouvaient difficilement apprendre l'anglais si la langue dans laquelle ils étaient instruits n'était pas le français ${ }^{100}$. En 1927, le gouvernement Ferguson s'inclina finalement et abolit le Règlement XVII.

\section{Eugénie Lorans, la crise de $\mathbf{1 9 2 9}$ et le déclin du mouvement de la bonne entente}

Son objectif premier ayant été atteint au milieu des années 1920 par l'abrogation du Règlement, le mouvement de la bonne entente connut une autre transformation. L'ancienne LBE prit une nouvelle forme sous la direction d'Eugé- 
nie Lorans, d'Ottawa, sa nouvelle présidente à partir de la fin de 1925. C'était la première fois que les activités du mouvement allaient être orientées par des femmes. Le premier comité de direction de Lorans, composé d'anglophones et de francophones, comportait cinq autres femmes mariées, ainsi qu'une fonctionnaire et une enseignante. Il y avait également quatre hommes - deux fonctionnaires et deux avocats. L'ensemble des membres du comité de direction étaient originaires d'Ottawa. La plupart, sinon tous, n'avaient jamais fait partie de l'ancienne Ligue. Ils avaient néanmoins été inspirés par son objectif de « réaliser l'union des races francophone et anglophone au Canada » et de " promouvoir un esprit de patriotisme et d'équité ${ }^{101} »$.

Sous la direction de Lorans, la Ligue s'éloigna de sa forme originelle de mouvement interprovincial et de groupe de pression, pour devenir un important club social municipal. Elle continua à produire des documents pédagogiques destinés à " planter la graine de l'unité au sein des masses ${ }^{102}$ ". Mais un examen plus approfondi des activités de la Ligue à la fin de la décennie donne à penser qu'elle s'était écartée des questions concrètes et litigieuses auxquelles elle s'était attaquée pendant les années de la BUA et de l'ULO. La ligue se transforma plutôt en club de loisirs - elle devint une tribune pour des conférences agréables sur l'histoire et la culture, ponctuées de concerts ou de présentations sur la littérature. Toutes ces activités avaient pour but de mettre en lumière la précieuse contribution à l'histoire et à la culture du pays des deux solitudes du Canada ${ }^{103}$.

Les changements apportés aux activités et aux champs d'intérêt de la Ligue sont compréhensibles eu égard au contexte. Le problème du Règlement XVII était essentiellement un conflit entre Ontariens - c'était un problème qui concernait la province. Il n'était que naturel que l'ULO, qui était 
ontarienne (contrairement à la LBE, qui était implantée en Ontario et au Québec et avait une vision interprovinciale), se soit chargée de faire pression sur les politiciens ontariens. De plus, le Règlement fut abrogé à peu près au moment où Lorans fut nommée à la présidence. Sans cette cause célèbre, il était normal que le mouvement perde de sa pertinence. De plus, il n'était plus nécessaire de faire pression sur le gouvernement pour qu'il favorise le rapprochement des élites. Les libéraux portés au pouvoir en 1921, principalement grâce au soutien dont ils jouissaient au Québec, se montrèrent beaucoup plus réceptifs aux préoccupations de cette province. Alors que Borden avait dirigé un "gouvernement de coalition " pratiquement sans apport du Canada français, Mackenzie King prit soin de consulter son lieutenant au Québec, Ernest Lapointe, sur tous les sujets concernant sa province. Finalement, le déclin des pressions politiques en coulisses peut être partiellement attribué au fait que Lorans avait moins de possibilités que ses prédécesseurs masculins d'exercer ce genre de pressions. En s'efforçant de limiter les activités de la LBE et d'en faire des tribunes sociales d'une échelle plus réduite, Lorans et son conseil exécutif à prédominance féminine faisaient de leur mieux avec les outils dont ils disposaient à l'époque.

Malgré son édulcoration - ou peut-être à cause d'elle le mouvement conserva parmi ses membres un vaste éventail de figures politiques, notamment des gouverneurs généraux, des premiers ministres provinciaux et fédéraux passés et présents, des chefs de l'opposition, des ministres fédéraux et provinciaux, des députés fédéraux, des députés provinciaux et des sénateurs tant conservateurs que libéraux ${ }^{104}$. Il semble à tout le moins raisonnable de conjecturer que la Ligue a contribué à favoriser une atmosphère plus conciliante parmi les cercles très influents d'Ottawa. Mais, en 1930, elle était net- 
tement devenue partisane. Dans la correspondance échangée entre Lorans et le chef de l'opposition de l'époque, Mackenzie King, elle se lamente sur les déboires électoraux des libéraux. Lorans va jusqu'à offrir que la Ligue serve à l'avenir d'outil de propagande pour le Parti libéral ${ }^{105}$. Alors que la crise de 1929 s'éternisait, la Ligue connut d'autres transformations et commença à se consacrer à l'aide aux chômeurs ${ }^{106}$. On ne pouvait guère demander à la plupart des Canadiens des années de famine de l'époque de s'intéresser à la cause altruiste de l'unité nationale. Ayant perdu de'sa pertinence, la Ligue fut lentement éclipsée par des préoccupations plus pressantes $^{107}$.

\section{Estimation de l'importance globale du mouvement de la bonne entente}

Lancé en grande pompe, à un moment où l'unité nationale était la question intérieure la plus urgente à régler, le mouvement de la bonne entente, lorsqu'il se termina, n'était plus que l'ombre de lui-même. Il est difficile de mesurer son importance globale de 1916 jusque dans les années 1930. En fait, cela exigerait une analyse systématique approfondie qui va bien au-delà des moyens et des objectifs du présent article. La question est toutefois importante et mérite d'être au moins abordée ici.

À ses débuts en 1916, le mouvement semble avoir eu droit à une abondante et optimiste couverture médiatique, tant dans la presse anglophone que francophone. Godfrey, Mulloy et Hawkes y avaient veillé grâce à leurs visites de relations publiques auprès d'importants propriétaires et rédacteurs en chef de journaux de l'époque. La rencontre de Mulloy avec l'influent O. S. Perrault, "l'administrateur et le directeur de l'Imperial Tobacco Company, qui entretenait d'étroites re- 
lations avec d'autres intérêts commerciaux et financiers ${ }^{108}$ ", par exemple, s'avéra particulièrement mémorable :

Perrault a passé la soirée dans ma chambre hier jusqu'au petit matin. Je n'avais encore jamais rencontré un homme qui pouvait ingurgiter autant de scotch et en ressentir si peu d'effets discernables. À propos, nous avons appris à bien nous connaitre et, étant donné toutes les personnes qu'il connait dans le milieu journalistique - sa compagnie dépense des milliers de dollars en publicité chaque année - je crois qu'il sera très utile ${ }^{109}$.

Néanmoins, Mulloy ne se limita pas à courtiser des alliés. Il s'aventura également en "territoire ennemi » lorsqu'il rendit visite au nationaliste Henri Bourassa, rédacteur en chef du journal Le Devoir, en août 1916. Il semble que Hawkes avait tenté de persuader Bourassa de devenir membre, mais sans grand succès. Au premier abord, cela peut étonner, étant donné la similitude apparente entre l'égalité des droits des anglophones et des francophones au Canada défendue par Bourassa d'un côté, et de l'autre l'idéal de la bonne entente. Mais Bourassa considérait avec scepticisme une entreprise dont les initiateurs étaient impliqués dans la National Service League $^{110}$. Néanmoins, l'ancien impérialiste Mulloy a été surpris d'avoir passé un aussi bon moment avec son soi-disant adversaire. "Je dois avouer que ce furent sans doute les deux heures les plus agréables de mon voyage au Québec....Bourassa, courtois, franc et cordial, alluma sa pipe et nous avons commencé. L'atmosphère fut du début à la fin empreinte de cordialité et de camaraderie. " Il semble que Mulloy ait fait une tout aussi bonne impression sur Bourassa : «Il m'est toujours plus facile de m'entendre avec un véritable impérialiste qu'avec un faux patriote ", déclara-t-il à son invité. Les deux hommes eurent une longue conversation sur un vaste éventail de sujets, ainsi que sur la guerre et sur la participation du Canada en particulier ${ }^{111}$. 
Les appréhensions de Bourassa l'empêchèrent finalement d'adhérer au mouvement. Toutefois, il chargea un de ses rédacteurs du journal Le Devoir, Ernest Bilodeau, d'assister aux débats des conférences d'octobre 1916 et 1917 et d'en faire le compte rendu. Le journal publia de nombreux articles sur les réunions, mais leur ton était détaché. Bilodeau attira l'attention sur l'extravagance et la somptuosité des banquets et s'amusa du fait que les participants anglophones et francophones ne se mélangeaient guère. Il s'intéressa particulièrement aux discours nationalistes de Lamarche et de Dandurand, soulignant que le Canada était plus important pour les francophones que l'Empire ${ }^{112}$. Les discours des recruteurs, comme Guthrie, ou de ceux qui s'étendaient en long et en large sur le devoir envers l'Empire, comme Hearst, étaient encore largement entachés de suspicion. Bilodeau était néanmoins impressionné par la sincérité manifeste d'un certain nombre de participants anglophones, tout particulièrement de Mulloy qui ne mâchait pas ses mots. L'hospitalité dont on fit preuve envers les Québécois au cours de leur séjour en Ontario fit également bonne impression sur l'ancien sceptique qu'était Bilodeau : «Les Ontariens de la Bonne-Entente ont fait preuve d'une indiscutable sincérité dans leur désir de mettre fin à la désunion qui sépare les deux grandes races canadiennes, nous n'avons point de doute là-dessus. Et leur sincérité s'est manifestée en une admirable hospitalité. On ne peut que leur souhaiter la pleine réussite de leurs désirs ${ }^{113}$. »

Au début, les articles du journal davantage grand public qu'était La Presse étaient d'un optimisme absolu, comme en témoigne le reportage sur l'ouverture de la convention à Montréal, le 10 octobre 1916 :

Il est certain [...] que les deux grandes races [...] ont fait un grand pas dans la voie de la conciliation et dans le règlement pacifique des questions épineuses qui les sé- 
paraient jusqu'ici. On a aboli pour la circonstance toute distinction de langue et de religion pour ne voir que des Canadiens animés des mêmes sentiments quand il s'agit de la conservation, de l'agrandissement et de l'exaltation de la patrie canadienne ${ }^{114}$.

Au départ, on sent un véritable espoir que le mouvement va créer au moins un semblant de rapprochement, fera disparaître les propos les plus agressifs et, peut-être même, résoudra le problème du recrutement et des droits linguistiques de la minorité. Ce dernier point fut davantage traité dans les journaux francophones et le premier dans la presse anglophone.

À la fin de la guerre, cependant, de nombreux francophones exprimèrent un scepticisme reflétant celui des bonneententistes désillusionnés. Un éditorial du journal Le Droit, intitulé « La Question du Québec », accueillait d'un ton critique compréhensible la création de la BUA :

Nous rencontrons, écoutons, lisons assez souvent des 'bonnes ententistes' de langue anglaise, apparemment animés des meilleures dispositions à notre égard [....] Ils nous parlent avec de grands gestes et des sourires engageants des bonnes relations qui devraient exister entre les deux grandes provinces-sœurs, entre les deux grandes races qui habitent ce dominion; mais ils ne mettent jamais le doigt sur la place ${ }^{115}$.

Ce scepticisme se manifestait également dans des lettres envoyées par le public à Mikel «L'Association de Bonne Entente, écrivait J. Edmond Cloutier, secrétaire de l'Association d'Éducation d'Ontario, a tenu de beaux discours sur l'entente et l'union des races; de résultats pratiques, de sympathie véritable de la part du peuple, elle n'en a obtenue aucuns (sic). Au contraire, celui-ci l'a décriée ${ }^{116}$."

Suite aux désillusions provoquées par la conscription et le gouvernement de coalition, on ne pouvait guère attendre 
d'organes nationalistes comme Le Devoir de Bourassa, et L'action française de Lionel Groulx, qu'ils aient confiance dans les efforts de Mikel pour ressusciter le mouvement de la bonne entente. Seule la réunion de la BUA de septembre 1920 eut droit à une mention dans Le Devoir. Ce bref article n'était guère enthousiaste en comparaison du commentaire de Bilodeau de janvier $1917^{117}$. Pour sa part, L'Action française, lancée en janvier 1917, ne fit pratiquement aucune mention de l'ancienne LBE, ni de la BUA qui lui succéda ${ }^{118}$. Au lieu de discuter de la nécessité de trouver un moyen de permettre à l'Ontario et au Québec de coexister, le journal tenait plutôt à protéger les Canadiens français contre «le poison colonial anglo-saxon ${ }^{119}$ ». À peu près au moment ou la BUA tint sa première réunion, au milieu de 1918, Groulx s'insurgeait contre l'idée que «la Confédération canadienne était une chose nécessairement éternelle ${ }^{120} \%$. Alors que même Le Devoir fit une place dans ses pages à la troisième réunion de la « Meilleure Entente ", les rédacteurs de L'Action française souhaitaient davantage promouvoir l'idée de créer un État séparé où les francophones pourraient une bonne fois pour toutes fuir «le fanatisme qui caractérise nos voisins d'Ontario ${ }^{121} »$. Le journal qualifia les initiatives ultérieures de bonne entente d'hypocrites et d'inutiles ${ }^{122}$.

Pour leur part, les journaux anglophones d'Ontario manifestèrent une tendance à la modération de ton suite aux initiatives ultérieures de bonne entente. Le premier congrès de la BUA de Mikel, en juillet 1918, eut droit à une couverture positive dans l'Intelligencer de Belleville, et le Daily Ontario. Un éditorial du Daily Ontario, par exemple, reprenait une phrase de l'ancien maire de Montréal, Louis-Arsène Lavallée, lorsqu'il demanda si la Suisse était «moins unie parce qu'elle tolère et reconnaît officiellement trois langues à l'intérieur de ses frontières ». Les deux langues du Canada, poursuivait-il, 
font partie intégrante de l'histoire du pays. « La véritable force de désintégration dans notre dominion est la promotion persistante de préjugés concernant la race et les croyances par des politiciens sans envergure et des journaux irresponsables. » Le journal admonestait les Ontariens qui «parlaient de faire rentrer de force l'anglais dans la gorge des habitants du Qué$\operatorname{bec}^{123}$ ». Dans la même veine, le Border Cities Star de Windsor et l'Evening Examiner de Peterborough réagirent au congrès de la LBE de janvier 1925 en demandant l'abrogation du Règlement XVII comme preuve de la sincérité de l'Ontario. Les journaux francophones tant de l'Ontario que du Québec le remarquèrent. «Ceux qui ont suivi de près la presse ontarienne depuis douze ans savent très bien que les opinions émises dans cette presse n'ont pas l'habitude de ressembler à celles que nous venons de lire ", déclarait avec optimisme L'Action catholique $^{124}$.

L'historien Patrice A. Dutil a suggéré que les bonneententistes les plus sincères ont « doucement injecté la colle qui a finalement cimenté le pays ${ }^{125} »$. Le mouvement avait du moins permis d'instaurer des rapports positifs entre les leaders des deux provinces, ce qui n'était pas le cas auparavant. Même Cameron admettait que "la bonne entente offre aux Ontariens une rare occasion de côtoyer des Canadiens français, ce qui ne peut qu'avoir eu une incidence positive sur les préjugés existants ${ }^{126} \%$. On pouvait en dire tout autant des répercussions de la couverture médiatique bienveillante à laquelle le mouvement eut droit à ses débuts, et occasionnellement par la suite. En fait, l'importance des personnalités politiques directement impliquées dans le mouvement, ou qui, à tout le moins, en embrassaient les idées, lui garantissait une certaine attention favorable dans les journaux. Cette couverture médiatique ainsi que les diverses activités pédagogiques et de propagande organisées par le mouvement durant toute 
son existence ont rejoint une partie du public, soit directement, soit par le bouche à oreille ${ }^{127}$. Godfrey se rappelait comment l'équipe de la bonne entente avait réussi à récolter 800 $000 \$$ pour des fonds patriotiques en temps de guerre auprès de quelque 106000 « travailleurs » montréalais, la plupart francophones $^{128}$. Il est impossible de dire combien des quatre cent mille membres de l'Association fraternelle canadienne adhéraient à ses politiques officielles de rapprochement ${ }^{129}$. De plus, l'aval de l'Association du barreau canadien aura été important, particulièrement dans un pays dont l'élite et les classes politiques étaient principalement constituées d'avocats. Le discours sur la bonne entente a pu aussi servir à modérer les politiques et les discours des personnalités publiques qui ne faisaient même pas partie du mouvement. Après tout, c'est par ces voies que le Règlement XVII fut finalement abrogé.

Il demeure toutefois impossible de savoir avec certitude quelles sont les activités de bonne entente qui ont influencé l'opinion publique et l'atmosphère politique en général, ni si les divisions auraient été, ou non, exacerbées sans l'influence modératrice du mouvement. En fin de compte, le pays se divisa en fonction des groupes linguistiques lors des élections de 1917, le gouvernement d'u union " imposa par la suite la conscription, les animosités persistèrent et les Canadiens français de l'Ontario durent attendre quinze longues années pour voir disparaître le Règlement honni. Sans le mouvement de la bonne entente, les choses auraient peut-être été pires. Mais on était loin de la société unie à laquelle les fondateurs de la première Ligue de la Bonne Entente avaient rêvé.

Le mouvement eut, à tout le moins, une importance symbolique pour ses créateurs. À cet égard, l'optimisme quelque peu naif dont fit preuve Georges Garneau durant les premières années de la bonne entente est typique : 
Messieurs, je ne peux me retenir de vous dire que vous avez écrit une page d'histoire à Hamilton aujourd'hui... Vous, juge Pelletier, avez parlé d'un livre d'or dans lequel vous voudriez voir écrit les noms de ces hommes qui ont été les pères de ce mouvement et que ne devraient jamais oublier leurs concitoyens reconnaissants. Le premier nom à inscrire dans ce livre sera le vôtre, Godfrey. (Applaudissements) C'est à vous, par votre amour pour votre pays, votre patriotisme, vos idéaux élevés, le courage et l'inébranlable détermination avec lesquels vous avez poursuivi les idéaux qui étaient les vôtres, le tact et le bon sens dont vous avez fait preuve en toute circonstance, que le mouvement doit son succès. (Applaudissements ${ }^{130}$ )

Certains des premiers participants au mouvement se voyaient comme des sortes de pères de la Confédération - les créateurs d'un mouvement audacieux qui apporterait au Canada la nationalité et l'unité qu'il méritait. Ce n'était nullement une coïncidence si les premières conférences de la LBE avaient été entourées de toute la pompe et de toute la solennité d'un immense exercice de construction de la nation. Il suffit d'un regard aux programmes élaborés, aux menus des dîners, aux coupures de presse conservées, aux histoires officielles et à la correspondance et aux discours transcrits pour se rendre compte des ambitions des membres des cinq premières années. Le fait même que Godfrey et Mikel ont préservé avec tant de soin les registres du mouvement montre bien l'importance historique qu'ils espéraient qu'il acquerrait avec le temps.

Lors de son dixième anniversaire, à Ottawa, le 14 mars 1928, la LBE remit à Eugénie Lorans un certificat de membre à vie. Le document est superbement peint et plein de symbolisme. Au centre s'élève un gros arbre aux profondes racines, avec sur ses branches droites et gauches deux grandes feuilles d'érable. Une de ces feuilles est ornée de la fleur de 
lys française et l'autre de la rose, du chardon et du trèfle des îles Britanniques. De chaque côté de la peinture est inscrit, en français et en anglais, la devise : "Se comprendre et s'aimer pour s'entr'aider » - «To Love to Help and to Understand each Other ${ }^{131}$. » Pas un mot de la cérémonie dans les journaux d'Ottawa. Les éditorialistes écrivaient plutôt sur l'extrémisme des orangistes et sur l'animosité que soulevait encore la bataille pour les droits des francophones en Ontario ${ }^{132}$.

\section{$* * *$}

De sa création à sa disparition, le mouvement de la bonne entente fit entendre, du moins jusqu'à un certain point, la voix de la modération et de l'unité au sein d'une société par ailleurs divisée. Quelles qu'aient été les lacunes du mouvement dans ses premières années, l'appel au rapprochement et à la réconciliation offrit au moins une solution de rechange à la rhétorique préjudiciable de l'animosité, particulièrement en Ontario. C'est cette animosité qui pendant la Grande Guerre poussa des hommes plus modérés à l'action. Le mouvement n'a peut-être pas empêché les divisions linguistiques de s'exacerber au pays, mais il a au moins posé les fondations des efforts de réconciliation de l'après-guerre. En fait, nombre de ses premiers fondateurs - et notamment des impérialistes comme John Godfrey et Lorne Mulloy - avaient été tellement touchés par leurs premières expériences qu'ils participèrent à la résurrection du mouvement à la fin de la guerre. C'est ce deuxième effort pendant l'après-guerre qui fit connaitre aux politiciens les doléances des Canadiens français et, en fin de compte, aboutit à l'abolition du Règlement XVII en Ontario. À une époque où le pays était profondément divisé, l'idéal de la bonne entente favorisa l'émergence d'une conscience morale. Même si, dans les dernières années de son existence, le mouvement perdit de sa pertinence, il continua, même si ce 
n'est que de façon limitée, à mettre en contact les anglophones et les francophones.

L'influence globale du mouvement de la bonne entente est, au mieux, difficile à déterminer. La question demeure donc: Où se situe ce mouvement au sein de l'histoire en général, particulièrement lorsqu'il semble avoir été à contre-courant de bien des tendances de son époque ? L'existence même du mouvement démontre aux historiens que le Canada anglophone et francophone n'était pas aussi monolithique qu'on le croit parfois. Bien que les voix les plus fortes dans la presse et en politique semblent avoir été celles de la division et de l'animosité, la bonne entente nous rappelle qu'il existait au moins un certain désir de rapprochement et de réconciliation. Les échecs du mouvement donnent à penser que c'était effectivement une exception à la règle. Mais ses succès en font également une force dont il vaut la peine de tenir compte dans l'histoire du Canada. De plus, la société canadienne de la première moitié du $\mathrm{XX}^{\mathrm{e}}$ siècle, et les relations entre francophones et anglophones en général, peuvent avoir été plus complexes que nous ne sommes souvent portés à le croire.

Dans un contexte plus général, les initiatives de bonne entente apparaissent comme des tentatives pour restaurer le rapprochement des élites qui avait caractérisé les gouvernements de Baldwin et Lafontaine, de Macdonald et Cartier, et de Laurier. Ce rapprochement des élites s'effondra sous le gouvernement Borden à cause des relations entre le Canada et l'Empire, et du nationalisme intolérant et raciste des Canadiens anglais, ce qui culmina dans la crise de la conscription ainsi que dans la discrimination continuelle envers les minorités francophones, particulièrement en Ontario. Le rapprochement des élites redevint possible lorsque le nationalisme anglo-canadien fut marginalisé en faveur d'un nationalisme civique canadien dans les années 1920 - nationalisme qui fut 
embrassé par le Parti libéral de Mackenzie King et son gouvernement. Cela fit beaucoup pour apaiser les nationalistes et les politiciens canadiens-français modérés comme Ernest Lapointe ${ }^{133}$. Une part du mérite d'avoir restauré le rapprochement des élites ainsi qu'un nationalisme civique canadien peut être attribué aux membres du mouvement de la bonne entente.

Traduction : Christian Bérubé

\section{NOTES}

${ }^{1}$ J'aimerais remercier le Conseil de recherches en sciences humaines et le Régime de bourses d'études supérieures de l'Ontario pour avoir assuré le financement qui a rendu cette étude possible. J'aimerais également remercier Michael D. Behiels et Peter Rider de leurs conseils et de leur encouragement au cours de la rédaction de cet article. Bien entendu, toutes les éventuelles erreurs ou inexactitudes de ce texte ne peuvent être attribuables qu'à son auteur.

${ }^{2}$ Elizabeth H. Armstrong, The Crisis of Quebec, 1914-1918, New York, Columbia University Press, 1937, p. vii. Dans cet article, pour faciliter la lecture, toutes les citations anglaises sont traduites en français.

${ }^{3}$ Carl Berger, dir., Imperialism and Nationalism, 1884-1914: A Conflict in Canadian Thought, Toronto, Copp Clark Publishing, 1969, p. 1 ; Carl Berger, The Sense of Power: Studies in the Ideas of Canadian Imperialism, 1867-1914, Toronto, University of Toronto Press, 1970, p. 259.

${ }^{4}$ Pour une comparaison plus complète entre Bourassa et les impérialistes canadiens les plus en vue, voir Sylvie Lacombe, La rencontre de deux peuples élus. Comparaison des ambitions nationale et impériale au Canada entre 1896 et 1920, Québec, Les Presses de l'Université Laval, 2002, 291 p. Voir également les textes de Bourassa dans Yvan Lamonde et Claude Corbo, dir., Le rouge et le bleu. Une anthologie de la pensée politique au Québec de la Conquête à la Révolution tranquille, Montréal, Les Presses de l’Université de Montréal, 1999, p. 301, 315.

${ }^{5}$ The Bonne Entente: How It Began, What It Has Done, and Its Immediate Programme, Toronto, Office of the Bonne Entente, 1917, p. 2. 
${ }^{6}$ Ici, je distingue la Ligue de la Bonne Entente du « mouvement de la bonne entente ». La première est une organisation, le second fait plus généralement référence à l'idéal de rapprochement entre Canadiens francophones et anglophones. Le terme « mouvement de la bonne entente » inclut toutes les initiatives et toutes les organisations créées en ayant en tête l'idéal de la « bonne entente », et ce de 1916 aux années 1930, dont la Ligue de la Bonne Entente (1916), la Better Understanding Association (1918) et la Unity League of Ontario (1921).

${ }^{7}$ Robert Craig Brown et Ramsay Cook, Canada, 1896-1921: A Nation Transformed, Toronto, McClelland and Stewart, Ltd., 1981, p. 265.

${ }^{8}$ Yvan Lamonde, Histoire sociale des idées an Québec, 1896-1929, vol. II, Montréal, Éditions Fides, 2004, p. 37-43. Honnêtement, compte tenu de la complexité de la période couverte par Lamonde, le lecteur ne devrait pas s'attendre à beaucoup plus d'un paragraphe sur le mouvement de la bonne entente.

${ }^{9}$ Brian Cameron, "The Bonne Entente Movement, 1916-1917: From Cooperation to Conscription ", Journal of Canadian Studies, vol.13, $\mathrm{n}^{\circ} 2$ (été 1978), p. 53.

${ }^{10}$ Voir Robert Choquette, Language and Religion. A History of English-French Conflict in Ontario, Ottawa, Les Presses de l'Université d'Ottawa, 1975, p. 229 236 ; Gaétan Gervais, «Le Règlement XVII (1912-1927) », Rerue du Nouvel Ontario, n 18 (1996), p. 175-186 ; Pierre Savard, «Relations avec Québec », dans Cornelius J. Jaenen, dir., Les Franco-Ontariens, Ottawa, Les Presses de l'Université d'Ottawa, 1993, p. 239-240.

${ }^{11}$ Robert Rumilly, Histoire de la province de Québec, vol. XXI, Montréal, Montréal-Éditions, 1940, p. 185.

${ }^{12}$ Robert Rumilly, Histoire de la province de Québec, vol. XXVI Montréal, Les Éditions Chantecler, Ltée, 1940, p. 260.

${ }^{13}$ The Bonne Entente, p. 1-2.

${ }^{14}$ François Charbonneau, La crise de la conscription pendant la Seconde Guerre mondiale et l'identité canadienne-française, Thèse de maîtrise (science politique), Université d'Ottawa, 2000, p. 36, 38.

${ }^{15}$ Le Devoir, 5 août 1914, p. 2.

${ }^{16}$ Charbonneau, La crise de la conscription, p. 36, 38. 
${ }^{17}$ Armstrong, The Crisis of Quebec, p. 85. Voir également Jean-Pierre Gagnon, Le 22e bataillon (canadien-francais) 1914-1919. Etude socio-militaire, Ottawa, Les Presses de l'Université Laval, 1986, p. 27-32.

${ }^{18}$ Jean Provencher, Québec sous la loi des mesures de guerre, 1918, Montréal, Éditions du Boréal Express, 1971, p. 23.

${ }^{19}$ Charbonneau, La crise de la conscription, p. 36-37.

${ }^{20}$ Dans Robert Lahaise, La fin d'un Québec traditionnel, 1914-1939, Montréal, Hexagone, 1994, p. 47.

${ }^{21}$ Dans J. L. Granatstein et J. M. Hitsman, Broken Promises: a History of Conscription in Canada, Toronto, Oxford University Press, 1977, p. 67.

${ }^{22}$ Procès-verbal de la première réunion de la Better Understanding Association, 25 juillet 1918, p. 6-7, Bibliothèque et Archives Canada (BAC), fonds de la Canadian Fraternal Association (ci-après FCFA), MG28-I3.

${ }^{23}$ The Bonne Entente, p. 1-2.

${ }^{24}$ Ibid., p. 2.

${ }^{25}$ Rumilly, Histoire de la province de Québec, vol. XXI, p. 181.

${ }^{26}$ Voir John M. Godfrey, « History of the Bonne Entente Including a Narrative of the Evidence Leading up thereto », 1917, p. 7-11, BAC, Fonds John Milton Godfrey (ci-après FG), MG30-C11 vol. 2 ; et Godfrey à Lord Shaughnessy, 20 juin 1916, BAC, FG, MG30-C11 vol. 1.

${ }^{27}$ The Bonne Entente, p. 3.

${ }^{28}$ Cette information était tirée de The Bonne Entente, p. 1-8 ; Godfrey, « History of the Bonne Entente », p. 1-22, BAC, FG, MG30-C11, vol. 2 ; La Presse, 10 octobre 1916, coupures de journaux, dans BAC, FG, MG30-C11, vol. 2 ; et le procès-verbal de la deuxième réunion de la Ligue de la Bonne Entente, 8-10 janvier 1917, p. 6-7, BAC, FG, MG30-C11, vol. 2.

${ }^{29}$ The Bonne Entente, p. 4-5.

${ }^{30}$ Voir, par exemple, le menu de la conférence de Sherbrooke, 11 octobre 1916, dans BAC, FG, MG30-C11, vol. 2.

${ }^{31}$ The Bonne Entente, p. 8.

${ }^{32}$ Voir, par exemple, le menu de la conférence de Niagara, 10 janvier 1917 , dans BAC, FG, MG30-C11, vol. 2. 
${ }^{33}$ Godfrey, « History of the Bonne Entente », p. 15, BAC, FG', MG30-C11, vol. 2.

${ }^{34}$ Georges Garneau, discours dans le procès-verbal de la deuxième réunion de la Ligue de la Bonne Entente, 8-10 janvier 1917, p. 110, 140, BAC, FG, MG30C11, vol. 2.

${ }^{35}$ Voir, par exemple, les discours prononcés par les colonels McCullough, de Hamilton, et Girouard, de Québec, dans le procès-verbal de la deuxième réunion de la Ligue de la Bonne Entente, 8-10 janvier 1917, p. 101, 231, BAC, FG, MG30-C11, vol. 2.

${ }^{36}$ Lomer Gouin, discours dans le procès-verbal de la deuxième réunion de la Ligue de la Bonne Entente, 8-10 janvier 1917, p. 60, BAC, FG, MG30-C11, vol. 2.

${ }^{37} \mathrm{~W}$. H. Hearst, discours dans le procès-verbal de la deuxième réunion de la Ligue de la Bonne Entente, 8-10 janvier 1917, p. 82, BAC, FG, MG30-C11, vol. 2.

${ }^{38}$ United we stand, divided we fall: address to our guests from Ontario by the Citizens of Three Rivers, 10 octobre 1916, p. 1, BAC, FG, MG30-C11, vol. 2.

${ }^{39}$ R. W. Leonard à Godfrey, 21 juillet 1916, BAC, FG, MG30-C11, vol. 1.

${ }^{40}$ Godfrey à Van Felson, 3 juillet 1916, BAC, FG, MG30-C11, vol. 1 .

${ }^{41}$ Hawkes à Godfrey, 13 juillet 1916, BAC, FG, MG30-C11, vol. 1.

${ }^{42}$ « Je crois qu'avec sagesse et tact nous pouvons accomplir de grandes choses. La principale difficulté, selon moi, est actuellement l'attitude de la province de l'Ontario. » Godfrey à Van Felson, 15 juillet 1916, BAC, FG, MG30-C11, vol. 1.

${ }^{43}$ Godfrey, « History of the Bonne Entente », p. 15, BAC, FG, MG30-C11, vol. 2.

${ }^{44}$ Dans La Presse, 10 octobre 1916, p. 12.

${ }^{45}$ Raoul Dandurand, discours dans le procès-verbal de la deuxième réunion de la Ligue de la Bonne Entente, 8-10 janvier 1917, p. 225, BAC, FG, MG30C11, vol. 2.

${ }^{46}$ Squair à Godfrey, 23 juin 1916, BAC, FG, MG30-C11, vol. 1.

${ }^{47}$ Voir Henry James Morgan, dir., The Canadian Men and Women of the Time: $A$ Hand-book of Canadian Biography of Living Characters, deuxième édition, To- 
ronto, William Briggs, 1912, p. 1053 ; Godfrey, « History of the Bonne Entente ", p. 13, BAC, FG, MG30-C11, vol. 2 ; et Le Devoir, 10 octobre 1916, p. 2. ${ }^{48}$ Voir Godfrey, « History of the Bonne Entente », p. 17, BAC, FG, MG30C11, vol. 2 ; et Morgan, The Canadian Men and Women of the Time, p. 11891190.

${ }^{49}$ Voir Cameron, «The Bonne Entente Movement », p. 42.

${ }^{50}$ Hawkes à Laurier, 17 juin 1916, BAC, fonds Sir Wilfrid Laurier (ci-après FL), MG26-G, vol.699.

${ }^{51}$ John Boyd, discours dans le procès-verbal de la deuxième réunion de la Ligue de la Bonne Entente, 8-10 janvier 1917, p. 175, BAC, FG, MG30-C11, vol. 2.

${ }^{52}$ Dans Cameron, «The Bonne Entente Movement », p. 52.

${ }^{53}$ Voir Desmond Morton, A Military History of Canada, quatrième édition, Toronto, McClelland \& Stewart, 1999 [1985], p. 143.

${ }^{54}$ Cameron, "The Bonne Entente Movement », p. 52-53.

${ }^{55}$ Voir R. Matthew Bray, «A Conflict of Nationalisms: The Win the War and National Unity Convention, 1917 ", Journal of Canadian Studies, vol. 15, $\mathrm{n}^{\circ} 4$ (hiver 1980-1981), p. 18-30, pour un résumé plus complet de la Win-the-War and National Unity Convention. D'après Bray, "L'article de Cameron se méprend sur l'origine de l'idée de la bonne entente, et en conséquence sa description de la relation entre les mouvements de la bonne entente et Win-the-War repose sur des bases fausses. "Voir la note en bas de page 2, p. 28. Je serais d'avis comme Bray que Win-the-War et le mouvement de la bonne entente n'étaient pas « une seule et même chose ». Cameron a raison de faire observer qu'un certain nombre de membres anglophones sont partis en faveur de Win-the-War, mais il est exagéré de parler d'exode massif. La plupart des participants à Win-the-War n'avaient jamais participé au mouvement de la bonne entente, et plus du tiers n'étaient ni ontariens ni québécois.

${ }^{56}$ Dans Cameron, «The Bonne Entente Movement », p. 53.

${ }^{57}$ Dans What Quebec Wants: Reply of La Presse to a Question from Ontario for the English Speaking People of the Dominion, With a Foreword by John Boyd, Montréal, Librairie Beauchemin, 1918. Les commentaires de Boyd figurent également dans La Presse, 27 février 1918.

${ }^{58}$ Voir Cameron, « The Bonne Entente Movement », p. 52.

${ }^{59}$ Voir Le Droit, 2 septembre 1920, coupure de presse, BAC, FCFA, MG28-I3. 
${ }^{60}$ Mikel aux membres de l'AFC, 19 juin 1918, BAC, FCFA, MG28-I3.

${ }^{61}$ Mikel aux membres de l'AFC, 18 juillet 1918, BAC, FCFA, MG28-I3.

${ }^{62}$ Information tirée du procès-verbal de la première réunion de la Better Understanding Association, 25 juillet 1918, BAC, FCFA, MG28-I3, et Morgan, The Canadian Men and Women of the Time.

${ }^{63}$ Mikel aux membres de l'AFC, 19 juin 1918, BAC, FCFA, MG28-I3.

${ }^{64}$ Mikel, discours dans le procès-verbal de la première réunion de la Better Understanding Association, 25 juillet 1918, p. 17, BAC, FCFA, MG28-I3.

${ }^{65}$ Platt, dans Ibid, p. 17.

${ }^{66} \mathrm{~J}$. Foy, Ibid, p. 31.

${ }^{67}$ Voir Le Droit, 2 septembre 1920, coupure de presse, BAC, FCFA, MG28-I3.

${ }^{68}$ Mikel dans le procès-verbal de la première réunion de la Better Understanding Association, 25 juillet 1918, p. 120, BAC, FCFA, MG28-13.

${ }^{69}$ E.T. Essery, Ibid, p. 8.

${ }^{70}$ Mulloy, Ibid, p. 5, 9.

${ }^{71}$ Champagne, Ibid, p. 20.

${ }^{72}$ Edwards, Ibid, p. 43.

${ }^{73}$ Mulloy, Ibid, p. 8.

${ }^{74} \mathrm{Ibid}$, p. 87.

${ }^{75} \mathrm{Ibid}$, p. $95-96$.

${ }^{76}$ Bélanger, Ibid, p. 100.

${ }^{77}$ Ibid, p. 102, 117.

${ }^{78}$ "Minutes of the "Better Understanding" meeting held at Ottawa », 28 novembre 1918, p. 1-2, BAC, FCFA, MG28-I3.

${ }^{79}$ "Statement of the Case, as prepared by the Committee », p. 33-35, BAC, FCFA, MG28-I3.

${ }^{80}$ "Call for the Third Meeting", s.d., p. 19, BAC, FCFA, MG28-I3.

${ }^{81}$ Meighen à Mikel, le 18 août 1921, p. 37, BAC, FCFA, MG28-I3.

${ }^{82}$ «Call for the Third Meeting», s.d., p. 19, BAC, FCFA, MG28-I3. 
${ }^{83}$ Dates diverses, p. 37-43, BAC, FCFA, MG28-I3.

${ }^{84}$ Morgan, The Canadian Men and Women of the Time, p. 84.

${ }^{85}$ Voir Patrice A. Dutil, «Against Isolationism: Napoléon Belcourt, French Canada, and "La grande guerre" ", dans David Mackenzie, dir., Canada and the First World War: Essays in Honour of Robert Craig Brown, Toronto, University of Toronto Press, 2005, p. 96-137.

${ }^{86}$ Même le Globe le qualifiait ainsi. Voir Morgan, The Canadian Men and Women of the Time, p. 84.

${ }^{87}$ " Rapport des activités du comité exécutif, 12 et 13 avril 1923 », Centre de recherche en civilisation canadienne-française, Université d'Ottawa (ci-après CRCCF), Fonds de l'Association canadienne-française de l'Ontario (ci-après FACFO), C2/21/6.

${ }^{88}$ Ibid, p. 35-36. Comme l'explique Choquette, « Les hommes responsables du retour à la normalité des relations entre francophones et anglophones entre 1918 et 1927 furent le sénateur libéral Napoléon Belcourt et les membres de la Unity League of Ontario. [... ] Cette ligue fut l'instrument principal du changement de l'opinion publique en Ontario pendant les années 1920. » Dans Robert Choquette, "Linguistic and Ethnic Factors in the French-Irish Catholic Relations in Ontario ", dans Canadian Catholic Historical Association Study Sessions, 39 (1972), p. 35-43.

89 « Rapport des activités du comité exécutif, 12 et 13 avril 1923 », p. 35-36, CRCCF, FACFO, C2/21/6. Voir également « Rapport du comite exécutif, 9 mai 1922 », p. 4-6, CRCCF, FACFO, C2/21/5.

${ }^{90}$ La Patrie, 31 janvier 1925, coupure de presse, CRCCF, FACFO, C2/257/8.

${ }^{91}$ L'Action catholique, 26 février 1925, coupure de presse, CRCCF, FACFO, C2/257/8.

${ }^{92}$ L'Action catholique, 6 février 1925, coupure de presse, CRCCF, FACFO, C2/ $257 / 8$.

${ }^{93}$ Cameron, "The Bonne Entente Movement », p. 53.

${ }^{94}$ Godfrey à Meighen, 12 janvier 1924, BAC, Fonds Meighen (ci-après FM), MG26-I, vol.100.

${ }^{95}$ Godfrey à Meighen, s.d. Réponse à une lettre de Meighen du 24 janvier 1924, BAC, FM, MG26-I, vol.100. 
${ }^{96}$ Godfrey à Meighen,12 janvier 1924, BAC, FM, MG26-I, vol.100. « Je vous écris ceci parce que je sais que vous avez une grande influence sur l'honorable Howard Ferguson, entre les mains duquel repose la solution de cette question extrêmement épineuse. »

${ }^{97}$ Godfrey à Meighen, s.d.. Réponse à une lettre de Meighen du 24 janvier 1924, BAC, FM, MG26-I, vol.100. Hawkes continua à prononcer des discours, rédiger des textes éducatifs et de propagande ainsi que des éditoriaux favorables au point de vue canadien-français.

${ }^{98}$ Note du 20 novembre 1925, BAC, Journaux de William Lyon Mackenzie King, consultés en ligne à http:// king.collectionscanada.ca (28 juillet 2007).

${ }^{99}$ En fait, Meighen semble avoir reçu beaucoup de courrier de la part d'autres bonne-ententistes, notamment des sénateurs Beaubien et Belcourt. Cette correspondance révèle qu'il était très inquiet du fait que le problème avait divisé le pays selon une fracture partisane, et ce, très au détriment de ses propres conservateurs au Québec. Voir, par exemple, Meighen à Godfrey, 24 janvier1924, BAC, FM, MG26-I, vol.100.

${ }^{100}$ Godfrey à Belcourt, 22 novembre 1923, CRCCF, FACFO, C2/97/10.

${ }^{101}$ Protocole d'entente de la Ligue de la Bonne Entente, 26 novembre 1925, p. 1, BAC, Fonds de la direction des corporations (ci-après FDC), RG95, vol.1172. Voir également la demande d'incorporation du 26 novembre 1925 dans le même dossier.

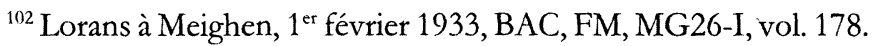

${ }^{103}$ Voir Lorans à Mackenzie King, 19 décembre 1930, BAC, Fonds Mackenzie King (ci-après FMK), MG26-J1, vol. 176.

${ }^{104}$ Ibid. Mackenzie King, Arthur Meighen, Rodolphe Lemieux, Éric Lapointe, Raoul Dandurand et les premiers ministres Taschereau et Ferguson, pour n'en nommer que quelques-uns.

${ }^{105}$ Ibid.

${ }^{106}$ BAC, MG26-I, Fonds Meighen, vol.178, bobine C3568, p. 111204. Lorans à Meighen, $1^{\text {er }}$ février 1933.

${ }^{107}$ Fait référence à diverses dates, 1927-1947, BAC, CBF , RG95, vol.1172. La ligue poursuivit modestement ses activités dans les années 1930 et au début des années 1940, mais lorsque Lorans, l'âme de l'organisation, mourut en 1942, elle cessa ses activités au bout de quelques années.

${ }^{108}$ Cameron, "The Bonne Entente Movement », p. 47. 
${ }^{109}$ Mulloy à Godfrey, 11 août 1916, BAC, FG, MG30-C11, vol. 1 .

${ }^{110}$ « Rien à faire avec Bourass[a]. » Hawkes à Godfrey, 17 juillet 1916, BAC, FG, MG30-C11, vol. 1. Voir également Mulloy à Godfrey, 2 août 1916, même volume.

${ }^{111}$ Mulloy à Godfrey, 11 août 1916, BAC, FG, MG30-C11, vol. 1 .

${ }^{112}$ Le Devoir, 10 octobre 1916, p. 1-2.

${ }^{113}$ Le Devoir, 15 janvier, p. 2.

${ }^{114}$ La Presse, 10 octobre 1916, p. 1.

${ }^{115}$ Le Droit, 16 mars 1918, p. 1.

${ }^{116}$ Cloutier à Mikel, 28 août 1920, p. 29, BAC, FCFA, MG28-I3.

${ }^{117}$ Le Devoir, 2 septembre 1920, p. 2.

${ }^{118} \mathrm{La}$ Bonne Entente est mentionnée en passant dans L'Action française, vol. 1, n², février 1917, p. 64.

${ }^{119}$ Ibid, p. 49.

${ }^{120}$ L'Action française, vol. 2, $\mathrm{n}^{\circ} 8$, août 1918, p. 347.

${ }^{121}$ L'Action française, vol. 4, n 9, septembre 1920, p. 405.

${ }^{122}$ Voir Rumilly, Histoire de la province de Québec, vol. XXVI, p. 258-259.

${ }^{123}$ Transcription du Daily Ontario, s.d., p. 12, BAC, FCFA, MG28-I3. Recoupement avec l'Intelligencer, 26 juillet 1918, coupure de presse, p. 10, BAC, FCFA, MG28-I3.

${ }^{124}$ Voir Le Nounel Ontario, 7 février 1925 et L'Action catholique, 26 février 1925, coupures de presse dans CRCCF, FACFO, C2/257/8.

${ }^{125}$ Dutil, « Against Isolationism », p. 128.

${ }^{126}$ Cameron, «The Bonne Entente Movement », p. 50.

${ }^{127}$ Voir, par exemple, «This bi-racial country », transcription d'une émission radiophonique de la chaîne anglaise de Radio-Canada par Jean I. Hunter, s.d., datant probablement du milieu des années 1930, CRCCF, FACFO, C2/257/ 8.

${ }^{128}$ Godfrey, "History of the Bonne Entente », 1917, p. 21-22, BAC, FG, MG30-C11, vol. 2.

${ }^{129}$ Mikel aux membres de la FAF, 18 juillet 1918, dans BAC, FCFA, MG28-I3. 
${ }^{130}$ Garneau, discours dans la transcription des délibérations de la deuxième réunion de la Ligue de la Bonne Entente, 8-10 janvier 1917, p. 110, 140, BAC, FG, MG30-C11, Vol. 2.

131 "Certificat de membre de la Ligue "Bonne Entente du Canada" (Inc.) ", 14 mars 1928, BAC, MG28-I339.

${ }^{132}$ Voir Le Droit, 14 mars 1928, et l'Ottawa Citizen, 14 mars 1928.

${ }^{133}$ L'auteur tient à remercier Michael D. Behiels pour cette analyse. 